\title{
A Multilevel Memetic Approach for Improving Graph k-Partitions
}

\author{
Una Benlic and Jin-Kao Hao \\ To appear in IEEE Transactions on Evolutionary Computation
}

15 March 2011

\begin{abstract}
Graph partitioning is one of the most studied NPcomplete problems. Given a graph $G=(V, E)$, the task is to partition the vertex set $V$ into $k$ disjoint subsets of about the same size, such that the number of edges with endpoints in different subsets is minimized. In this work, we present a highly effective multilevel memetic algorithm, which integrates a new multiparent crossover operator and a powerful perturbation-based tabu search algorithm. The proposed crossover operator tends to preserve the backbone with respect to a certain number of parent individuals, i.e. the grouping of vertices which is common to all parent individuals. Extensive experimental studies on numerous benchmark instances from the Graph Partitioning Archive show that the proposed approach, within a time limit ranging from several minutes to several hours, performs far better than any of the existing graph partitioning algorithm in terms of solution quality.
\end{abstract}

Index Terms-Graph partitioning, multi-parent crossover, tabu search, backbone, landscape analysis.

\section{INTRODUCTION}

Graph partitioning is one of the fundamental combinatorial optimization problems which is notable for its applicability to a wide range of domains, such as VLSI design [1], [39], data mining [47], image segmentation [37], etc. It is well known that the general graph partitioning problem is NP-complete [15], so approximate approaches are very useful to address this problem.

Evolutionary algorithms are among the most popular approaches for the graph partitioning problem. Some representative examples include: Mansour and Fox [29], who enforce the equi-partition constraint with a penalty term; Talbi and Bessiere [42], whose genetic algorithm is based on a cellular population structure; Bui and Moon [10], who additionally employ a preprocessing phase scheme that improves the space searching capability of the genetic algorithm; Gil et al. [16], who use the direct encoding for circuit partitioning; Kang and Moon [23], and Kim and Moon [27], who perform extensive experiments on graphs with up to 5,000 vertices that show an improvement over the local optimization approaches. In [43], von Laszewski employs a 'structural genetic operator' which copies subsets of vertices to the offspring. The current most effective population-based approach is the one reported by Soper et al. [38], which employs a multilevel heuristic algorithm to provide an effective crossover. Although this

Una Benlic and Jin-Kao Hao (Corresponding author) are with the LERIA, University of Angers, 2 Boulevard Lavoisier, 49045 Angers Cedex 01, France (e-mail: \{benlic,hao\}@info.univ-angers.fr) approach requires considerable computing time (up to one week), it achieves partitions significantly better than those generated by the state-of-art graph partitioning packages.

Moreover, a great number of well-known graph partitioning approaches are based on other popular metaheuristics including Tabu Search [12], [36], [4], Simmulated Annealing [21], Neural Networks [2], Swarm Intelligence [41], etc.

To handle very large graphs, the so-called multilevel paradigm has shown to be very effective for partitioning graphs [3], [19], [25], [44], [31]. The basic idea of multilevel approaches is to first coarsen the original graph $G$ down to a certain number of vertices, generate a partition of this much smaller graph, and then project this partition back towards $G$ by successively refining the partition.

In this paper, we introduce a new multilevel memetic algorithm which combines a dedicated multi-parent crossover operator based on the notion of backbone and a perturbationbased tabu search algorithm. This work extends thus a preliminary memetic algorithm presented in [5], where a different crossover operator and a hill-climbing based local search algorithm are used. Compared to this previous work, the new algorithm presented in this paper ensures better exploration with the new multi-parent crossover operator, and better exploitation provided by an effective tabu search algorithm. Furthermore, this paper additionally includes: (a) more extensive experimental evaluations on a set of benchmark instances from the Graph Partitioning Archive; (b) a detailed analysis on several key issues such as the distribution of local optima and the backbone size; (c) a comparison of the proposed crossover operator with the traditional uniform crossover, and analysis on the impact of perturbation within the proposed crossover operator; and (d) an analysis on the impact of the employed local search mechanism on the overall performance of our memetic algorithm.

The paper is organized as follows. In the next section, we recall the definition of the graph partitioning problem and some basic notations used. In Section III, we describe the multilevel paradigm, as well as the general scheme of the proposed multilevel approach. In Section IV, we present the memetic algorithm, which is the partition refinement mechanism of the multilevel approach. In Section V, we provide computational results of extensive experiments on benchmark instances from the Graph Partitioning Archive. In Sections VI, we show a landscape and backbone analysis, and based on the observations made, provide a motivation for our proposed multi-parent crossover operator. In Section VII, we compare the proposed 
crossover with a conventional uniform crossover operator, and study the impact of perturbation strength within our multiparent crossover operator. In Section VIII, we analyze the impact of local search on the overall algorithm performance before concluding in Section IX.

\section{PRoblem DESCRIPTION AND NOTATIONS}

Given an undirected graph $G=(V, E), V$ and $E$ being the set of vertices and edges respectively, and a fixed number $k$, a $k$-partition of $G$ can be defined as a mapping (partition function) $\pi: V \rightarrow\{1,2, \ldots, k\}$ that distributes the vertices of $V$ among $k$ disjoint subsets $S_{1} \cup S_{2} \cup \ldots \cup S_{k}=V$.

Let $\left\{S_{1}, S_{2}, \ldots, S_{k}\right\}$ be a partition of $V$ obtained by $\pi, E^{c}$ the set of all the cutting edges of $G$ induced by $\pi$, i.e. $E^{c}$ $=\left\{\{x, y\} \in E \mid x \in S_{i}\right.$ and $y \in S_{j}$ and $\left.i \neq j\right\}$, and let $\varphi$ be the set of all the partition functions of $G$. The graph $k$-partitioning problem consists in determining $\pi^{*} \in \varphi$ such that the partition $\left\{S_{1}, S_{2}, \ldots, S_{k}\right\}$ given by $\pi^{*}$ minimizes the number of cutting edges in $E^{c}$ while ensuring that each $S_{i}$, $i \in\{1,2, \ldots, k\}$ is of roughly equal size.

Throughout this paper, the initial input graph $G$ is supposed to have a unit cost weight for both vertices and edges. However, as explained in Section III-B, the multilevel approach generates intermediate (coarsened) graphs with weighted edges and vertices. It is then useful to define the notion of edge and vertex weight.

Let $|v|$ denote the weight of a vertex $v$ in a coarsened graph, which corresponds to the number of aggregated vertices of the initial graph. Then, the weight $W\left(S_{i}\right)$ of a vertex subset $S_{i}$ is equal to the sum of weights of the vertices in $S_{i}, W\left(S_{i}\right)=$ $\sum_{v \in S_{i}}|v|$. The weight of a set of edges in the coarsened graph can similarly be defined.

In this paper, we are essentially interested in finding almost evenly balanced partitions. The notion of balance is defined as follows. Let $W_{o p t}=\lceil|V| / k\rceil$ be the optimal subset weight, where $\lceil x\rceil$ represents the first integer $\geq x$, then the quantity $\varepsilon=\max _{i \in\{1 . . k\}} W\left(S_{i}\right) / W_{o p t}$ defines the degree of imbalance among the $k$ subsets of a partition $\left\{S_{1}, S_{2}, \ldots, S_{k}\right\} . \varepsilon=1$ means that the partition is perfectly balanced while $\varepsilon>1$ indicates an imbalanced partition with larger $\varepsilon$ corresponding to larger imbalance.

The optimization objective $f$ of our graph partitioning algorithm is to find a $k$-partition with the smallest number of edge cuts in $E^{c}$, such that each partition subset is of almost equal size $(\epsilon=1.00)$.

\section{Multilevel Memetic Algorithm FOR GRAPH PARTITIONING}

\section{A. General procedure}

Our multilevel memetic approach follows the general multilevel paradigm [9], [3], [19], [45]. Graph $G$ is first coarsened down to a certain number of vertices (coarsening phase), an initial partition of this much smaller graph is generated (initial partitioning phase), and then this partition is projected back towards the original graph (uncoarsening phase) followed by partition refinement.
The proposed multilevel approach, which employs memetic partition refinement at each uncoarsening step, is presented in Algorithm 1.

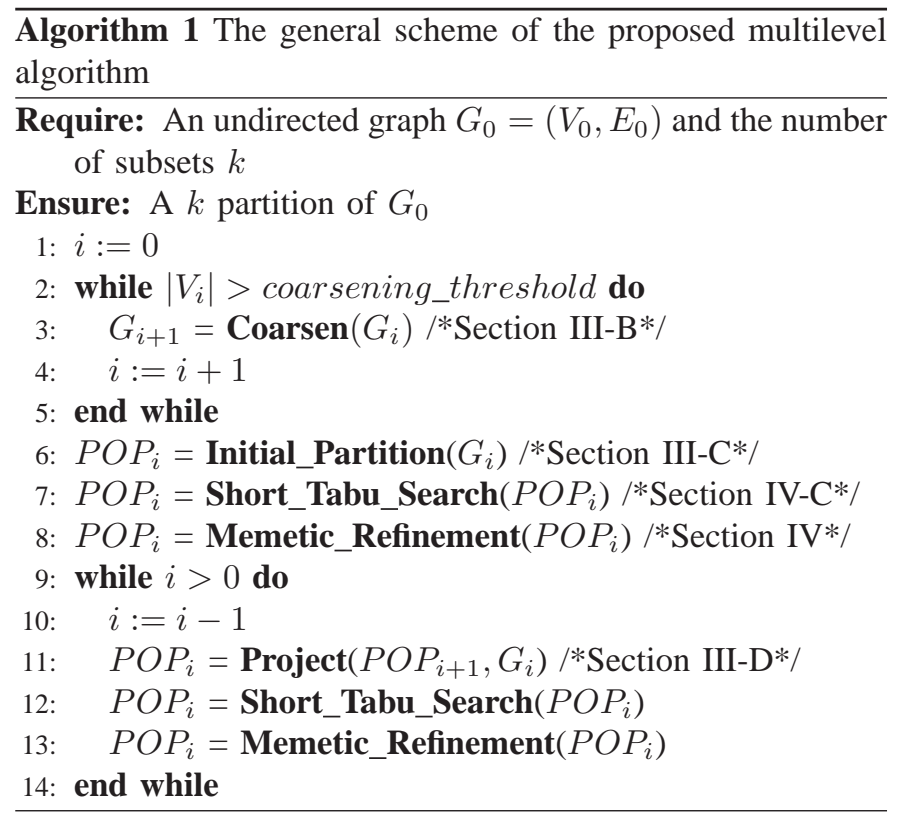

\section{B. Coarsening phase}

Let $G_{0}=\left(V_{0}, E_{0}\right)$ be the initial graph. Creating a coarser graph $G_{i+1}=\left(V_{i+1}, E_{i+1}\right)$ from $G_{i}=\left(V_{i}, E_{i}\right)$ consists in finding an independent subset of edges (matching) $\Gamma \subset E_{i}$, and then collapsing the two vertices of each edge in $\Gamma$ to form a new vertex in $V_{i+1}$. Any vertex that is not part of $\Gamma$ is simply copied over to $G_{i+1}$ (see Fig. 1 for an illustrative example).

When two vertices $v_{1}, v_{2} \in V_{i}$ are collapsed to form a new vertex $v_{a} \in V_{i+1}$, the weight of the resulting vertex $v_{a}$ is set equal to the sum of weights of vertices $v_{1}$ and $v_{2}$. Therefore, the weight of a vertex of a coarsened graph equals the number of aggregated vertices of the initial graph $G_{0}$.

Similarly, let $v_{a}, v_{b} \in V_{i+1}$ be two vertices formed by collapsing $\left\{v_{1}, v_{2}\right\} \in \Gamma$ and $\left\{v_{3}, v_{4}\right\} \in \Gamma$. All the edges incident to $\left\{v_{1}, v_{2}\right\}$ and $\left\{v_{3}, v_{4}\right\}$ are merged to form a new edge $\left\{v_{a}, v_{b}\right\} \in E_{i+1}$ with a weight that is set equal to the sum of weights of edges incident to $\left\{v_{1}, v_{2}\right\}$ and $\left\{v_{3}, v_{4}\right\}$.

One key issue here is the selection of the independent subset of graph edges $\Gamma$ to be collapsed at each step of the coarsening phase. This can be achieved by finding a maximal matching of the graph [32]. There exist polynomial time algorithms for tackling this problem, with running time of at least $O\left(|V|^{2.5}\right)$. Unfortunately, this is too slow to be applicable to the partitioning problem. That is why we compute an approximate maximal matching using a fast heuristic called heavy-edge matching (HEM), which has $O(|E|)$ time complexity [24]. This method considers vertices in random order, matching each unmatched vertex $v$ with its unmatched neighbor $u$, if any, such that the weight of edge $\{u, v\}$ is maximal among all the edges incident to $v$. An example of vertex and edge aggregation with HEM of an initial graph with seven vertices is provided in Figure 1. 


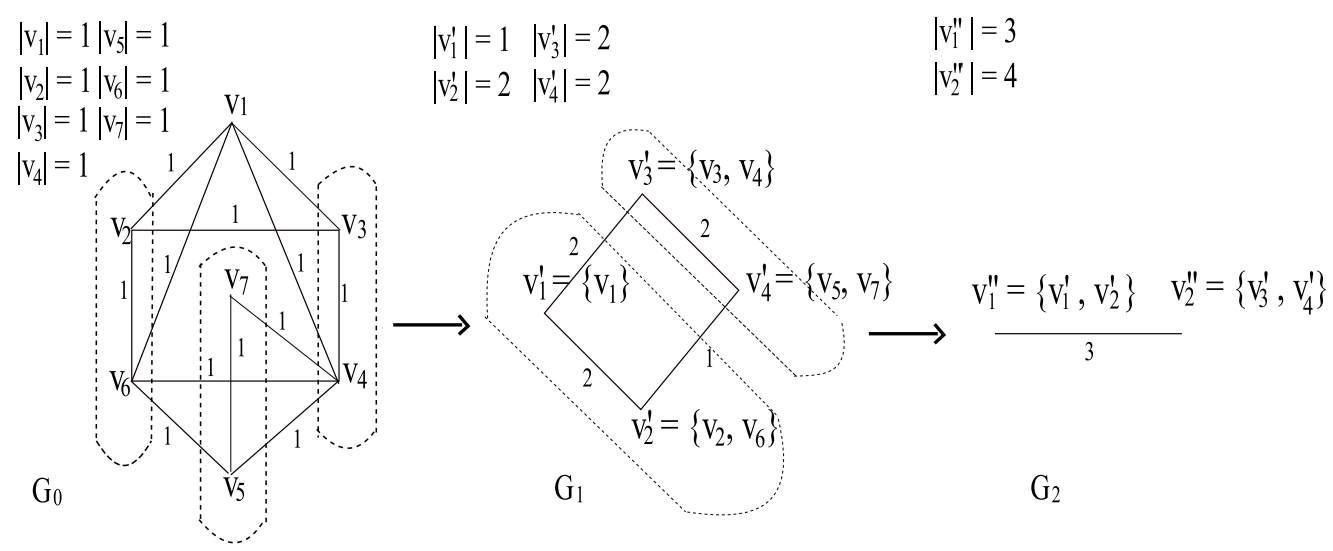

Fig. 1. An example of coarsening with HEM of an initial unweighted graph $G_{0}$ with seven vertices. The weight of a vertex $|v|$ of a coarsened graph $G_{i}$ equals the number of aggregated vertices of the initial graph $G_{0}$. The weight of the resulting edge, which is incident to the collapsed vertex $v_{c}=\{u, v\}$ is set equal to the sum of weights of all the edges incident to $u$ and $v$ minus the weight of edge $\{u, v\}$.

\section{Initial partition and its refinement}

To create each individual of the initial population in the second phase (line 6 of Alg. 1), we first assign randomly the vertices of the coarsest graph $G_{m}=\left(V_{m}, E_{m}\right)$ to subsets $S_{i} \in\left\{S_{1}, S_{2}, . . S_{k}\right\}$, such that each subset is as evenly balanced as possible, i.e. each $S_{i}, i \in\{1, \ldots, k\}$ has a similar weight $W\left(S_{i}\right)$. Afterwards, we apply a short run of the perturbation-based tabu search, previously presented in [6], to improve all individuals of this initial population (see Section IV-C), followed by the memetic refinement which is described in Section IV (lines 7-8 of alg. 1).

This refinement step is essential for our approach to improve progressively the quality of partitions. It should be noted that for certain graphs, it may be impossible to obtain a perfectly balanced initial partition, since weights of vertices in the coarsest graph are often greatly inhomogeneous. This imbalance is gradually reduced throughout each uncoarsening step, and (usually) completely eliminated by the end of the algorithm execution.

\section{Uncoarsening phase}

The uncoarsening phase carries out the inverse of the coarsening phase. The idea is to go from level to level, uncoarsening the clustered vertices in the same way they were grouped during the coarsening phase. The partition projection from a graph $G_{i}=\left(V_{i}, E_{i}\right)$ onto a partition of the parent graph $G_{i-1}=\left(V_{i-1}, E_{i-1}\right)$ is a trivial process. If a vertex $v \in V_{i}$ is in subset $S_{m}$, then the matched pair of vertices $v_{1}, v_{2} \in V_{i-1}$ which represents vertex $v \in V_{i}$ will also be in subset $S_{m}$.

Before projecting a partition on to the next level, we first apply a short run of the perturbation-based tabu search to improve all the individuals of the population, which is immediately followed by the memetic refinement (lines 1213 of alg. 1). Experiments show that the local optimization applied on the initial population before memetic refinement (lines 7 and 12 of Alg. 1) influences favorably the final result, though this influence is not very important.

As the uncoarsening-refining process proceeds, the partition quality of a graph $G_{i-1}$ is usually better than that of $G_{i}$ because there is a greater degree of freedom for refinement. This is one of the most attractive characteristics of a multilevel algorithm.

\section{THE MEMETIC REFINEMENT}

The general idea behind memetic approaches is to combine advantages of both crossover that discovers unexplored promising regions of the search space, and local search that finds good solutions by concentrating the search around these regions. Given an initial population which consists of locally optimal solutions, a memetic approach generates a new set of improved local optima by applying a crossover operator and/or mutation to the population, followed by local refinement. The success of this method depends critically on the crossover operator that discovers new promising regions of the search space by performing 'jumps' from one local optimum to another. These jumps need to be far enough to escape from the basin of attraction of the current local optimum, but still not too far to degenerate into a simple random search algorithm. Furthermore, in order to perform a directed 'jump', a crossover operator should be able to recognize what elements must be preserved through the recombination and what elements can be perturbed.

Motivated by the observation that high quality partitions share many groupings of vertices (backbone, see below), we propose in this work an original backbone-based multi-parent crossover (BBC) which preserves the backbone with respect to a certain number of parent individuals. After the offspring has been generated by the proposed crossover, it is refined with a perturbation-based tabu search algorithm. Finally, we apply a replacement strategy, which takes into consideration both the partition quality and the distance between individuals in the population.

The general architecture of our memetic approach is described in Algorithm IV. The main components are detailed in the following subsections.

\section{A. Encoding and fitness function}

Given a graph $G_{i}=\left(V_{i}, E_{i}\right)$ at level $i$ and an integer $k$, an individual $I$ corresponds to a partition of $V_{i}$ into $k$ disjoint 


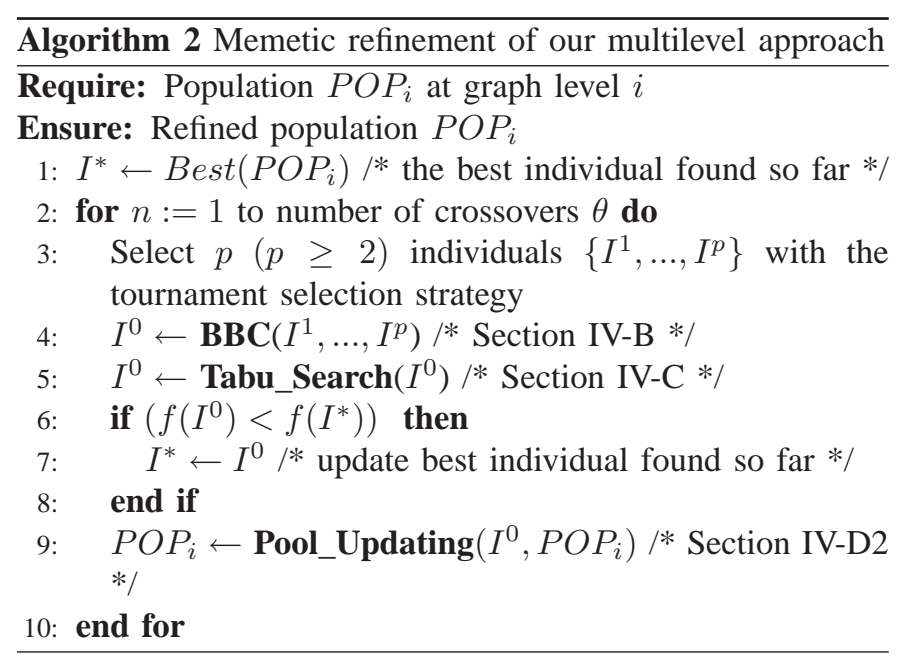

groups or subsets $I=\left\{S_{1}, \ldots, S_{k}\right\}$, such that each $S_{j}, j \in$ $\{1, \ldots, k\}$ is composed of vertices that are assigned to the $j^{t h}$ subset.

The optimization objective of our $k$-partitioning problem is to minimize the cutting edges in $E^{c}$ (see Section II), while maintaining the best possible balance between partition subsets.

The fitness function $f(I)$ of our memetic algorithm is directly related to the optimization objective and sums up the cutting edge weights of a $k$-partition (individual) $I=$ $\left\{S_{1}, \ldots, S_{k}\right\}$. More formally,

$$
\begin{gathered}
f(I)=\sum_{\{u, v\} \in E_{i}} \varrho_{u, v}(I) \\
\varrho_{u, v}(I)= \begin{cases}w_{u, v} & \text { if } u \in S_{x} \text { and } v \in S_{y}(x \neq y) ; \\
0, & \text { otherwise. }\end{cases}
\end{gathered}
$$

where $w_{u, v}$ represents the weight of edge $\{u, v\} \in E_{i}$, i.e. the number of unit cost edges of the original graph $G_{0}=\left(V_{0}, E_{0}\right)$ that are aggregated within $\{u, v\} \in E_{i}$ during the coarsening phase.

Then, individual $I^{A}$ is considered better than individual $I^{B}$ only if $f\left(I^{A}\right)<f\left(I^{B}\right)$ (lines 6-8 of algo. 2).

Since our goal is to find perfectly balanced partitions ( $\varepsilon=1.00)$, the partition balance is imposed as a constraint rather than an objective. However, as mentioned earlier, it is sometimes impossible to establish perfect balance in coarsened graphs since vertex weights can be extremely inhomogeneous. It is during the partition refinement of levels which are closer to the original graph that the balance condition is (usually) completely satisfied. More precisely, the tabu search procedure of our memetic algorithm employs two move operators that take care of partition imbalance by transferring vertices to subsets of smaller weight (see Section IV-C). In addition, the proposed backbone-based crossover operator insures that the balance is not degraded during the crossover process (see Section IV-B).

\section{B. Backbone and crossover}

1) Notion of backbone: Our backbone-based multi-parent crossover described in this section tries to preserve the back- bone with respect to a number of parent individuals while redistributing with a certain probability vertices that do not belong to the backbone.

For optimization problems, the term backbone is usually used to define a set of variables $B$ having the same value assignment throughout all the global optima, while the backbone size corresponds to the number of elements in $B$. The similar idea has been used in several contexts [13], [26], [46], [48].

For our graph partition problem, the notion of backbone can be defined as follows.

Definition 1 (Backbone): Let $G$ be a graph, $\Omega$ the set of all optimal $k$-partitions of $G$. The backbone $B$ of $G$ is a set of $k$ subsets of vertices $\left\{B_{1}, \ldots, B_{k}\right\}$ such that each $B_{i}$, $i \in\{1, \ldots, k\}$ is the subset of vertices that are grouped together throughout all the optima of $\Omega$.

Definition 2 (Backbone size): Given a backbone $B=$ $\left\{B_{1}, \ldots, B_{k}\right\}$, its size $|B|$ equals $\left|B_{1} \cup \ldots \cup B_{k}\right|$.

Such a definition cannot be applied in practice given that the optimal solutions are unknown (our goal is to find such a solution). Therefore, in this paper, we use a relaxed definition of backbone by considering a set of locally optimal (high quality) solutions. Therefore, if a set of vertices are shared through the set of selected $k$-partitions, these vertices are considered to have a high chance to be part of the backbone.

2) The backbone-based multi-parent crossover operator $(B B C)$ : Given the set $P=\left\{I^{1}, \ldots, I^{p}\right\}$ of $p$ parent individuals, BBC constructs the offspring $I^{0}=\left\{S_{1}^{0}, \ldots, S_{k}^{0}\right\}$ in $k$ passes (one for each subset of the partition). In each pass $\mu$ it performs the following steps:

1) Select a subset $S_{j}^{i}$ of $I^{i}$ such that the weight $W\left(S_{j}^{i}\right)$ is maximal across the subsets $j \in\{1 . . k\}$ of each individual $I^{i} \in P$, i.e. $\max _{i \in\{1 \ldots p\}, j \in\{1 . . k\}}\left\{W\left(S_{j}^{i}\right)\right\}$, with the constraint that at most $\lceil k / p\rceil$ subsets can be chosen from each individual $I^{i} \in P$ (line 5 of alg. 3).

2) Given $I^{i}$ and $S_{j}^{i}$ determined in Step 1, for each individual $I^{t} \in P(t \neq i)$, let $\prod_{t}$ contain the largest number of vertices that are shared by the subset $S_{j}^{i}$ of $I^{i}$ and a subset $S_{\eta}^{t}$ of $I^{t}$, i.e. $\prod_{t}=\left\{S_{j}^{i} \cap S_{\eta}^{t}\left|\max _{\eta \in\{1 . . k\}}\right| S_{j}^{i} \cap S_{\eta}^{t} \mid\right\}$. Then, $\Pi=\left\{\prod_{1}, . ., \prod_{p-1}\right\}$ forms a set of these vertex subsets (lines 6-9 of alg. 3).

3) Set $S_{\mu}^{0}=\prod_{1} \cap \prod_{2} \cap \ldots \cap \prod_{p-1} . S_{\mu}^{0}$ is the largest subset of vertices that are shared by all the parent individuals. For each vertex $v \in S_{j}^{i}$ and $v \neq S_{\mu}^{0}, v$ is assigned to subset $S_{\mu}^{0}$ of $I^{0}$ if $c(v) / p-1$ is greater than or equal to some random real number in the range $[0,1]$, where $c(v)$ is the number of subsets of $\prod$ in which $v$ occurs (lines $11-15$ of alg. 3).

4) When a vertex $v$ is assigned to subset $S_{\mu}^{0}$ of $I^{0}$ in the $\mu^{t h}$ pass, $v$ is removed from all the parent individual subsets in which it occurs, and the weights of these subsets are adjusted accordingly (lines $17-18$ of alg. 3 ).

After the previous four steps, the last step handles the unassigned vertices. Any vertex $v$ missing from $I^{0}$ is placed at random to a subset $S_{r}$ of $I^{0}$ such that $W\left(S_{r} \cup\{v\}\right) \leq W_{\text {opt }}$ (lines 21-26 of alg. 3), where $W_{\text {opt }}$ is defined in Section II. This step introduces a degree of diversification in the crossover 


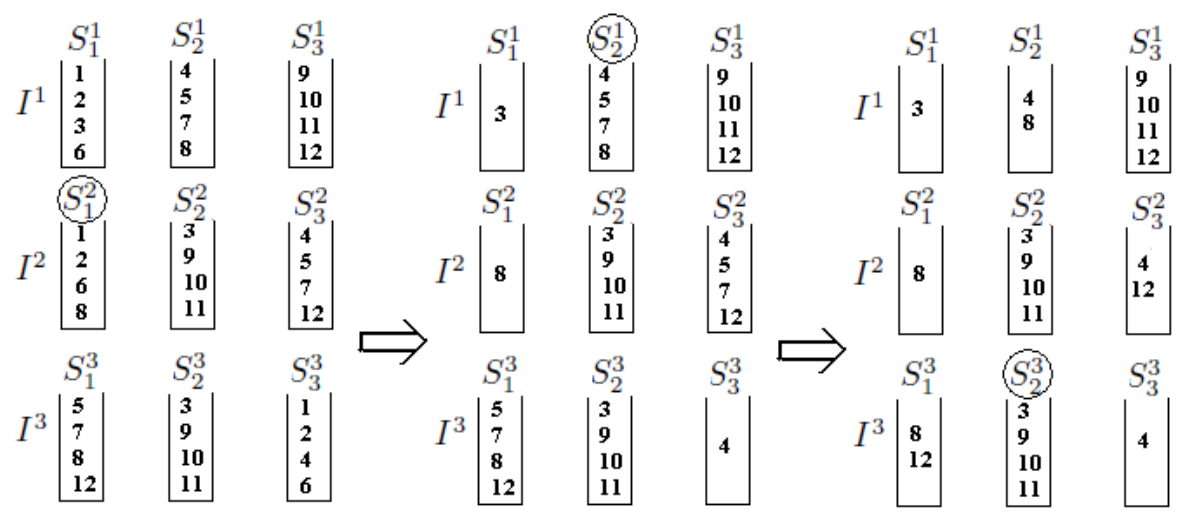

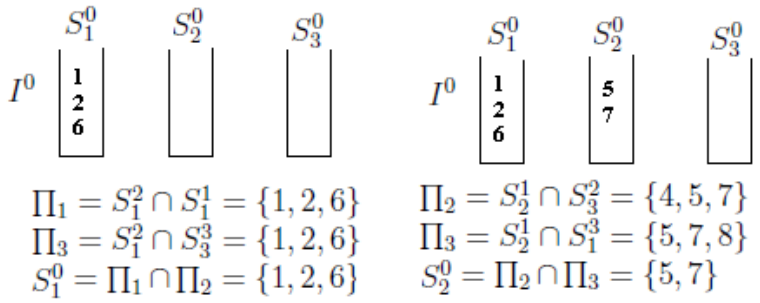

Pass 1
Pass 2 \{vertices 4 and 8 are assigned to $S_{2}^{0}$ with a probability of 0.5 .

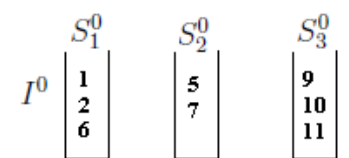

$\Pi_{1}=S_{2}^{3} \cap S_{3}^{1}=\{9,10,11\}$

$\Pi_{2}=S_{2}^{3} \cap S_{2}^{2}=\{3,9,10,11\}$

$S_{3}^{0}=\prod_{2} \cap \prod_{3}=\{5,7\}$

Pass 3 V Vertex 3 is assigned to $S_{3}^{0}$ with a probability of 0.5.$\}$

Fig. 2. An illustration of the BBC crossover with three parents. A circled subset of a parent corresponds to the subset chosen in the $\mu^{\text {th }}$ pass, i.e. the subset of maximal weight across all the parent individuals with the constraint that at most $\lceil k / p\rceil$ subsets can be chosen from each individual.

process.

Notice that the proposed BBC operator never degrades the balance with respect to the set of parent individuals $P$, since given a subset $S_{j}^{i}$ of individual $I^{i}$ which is chosen in the $\mu^{t h}$ pass (see line 5 of alg. 3), at most $\left|S_{j}^{i}\right|$ vertices can be transmitted to the subset $S_{\mu}^{0}$ of offspring $I^{0}$. In addition, an unassigned vertex $v$ in $I^{0}$ is assigned to a subset $S_{r}^{0}$ only if adding $v$ to $S_{r}^{0}$ does not exceed the expected optimal subset weight $W_{\text {opt }}$.

The complexity of the proposed crossover is $O\left(p * k *\left|V_{i}\right|\right)$, where $\left|V_{i}\right|$ is the number of vertices in graph $G_{i}\left(V_{i}, E_{i}\right)$.

To determine the subset $P \subset P O P$ of $p$ parent individuals, we employ the tournament selection strategy. Let $\lambda$ be the size of the tournament pool. We select each individual $I^{i} \in P$ in the following way: randomly choose $\lambda$ individuals from $P O P$; among the $\lambda$ chosen individuals, place the best one into $P$ if it is not already present in $P$.

An example of this crossover with three parent individuals $(p=3)$ for $k=3$ is provided in Figure 2.

\section{Perturbation-based Iterated Tabu Search improvement}

To improve the newly generated offspring, we apply an iterated tabu search algorithm [6] whose basic components are briefly described in this section.

Basically, the TS algorithm uses two neighborhood relations (call them $N_{1}$ and $N_{2}$ ) which are explored in a token-ring way. That is, we repeatedly apply one neighborhood search to the best local optimum produced by the other neighborhood. The algorithm incorporates as well a perturbation mechanism in order to bring diversification into the search.
1) Neighborhood relations: Given a subset $S_{i}$ of a $k$ partition $I=\left\{S_{1}, S_{2}, \ldots, S_{k}\right\}$, the basic idea of the neighborhood relations is to move a vertex $v$ from another subset to $S_{i}$. Such a move is constrained such that $v$ must be a border vertex relative to $S_{i}$, i.e. $v \notin S_{i}$ has at least one adjacent vertex in $S_{i}$. Note that in this way, the size of the neighborhoods is limited, since the set of border vertices relative to $S_{i}$ is generally of small size. In addition, such a neighborhood allows the search to concentrate around these critical vertices.

The key concept related to the two neighborhoods is the move gain, which represents the change in the optimization objective. It expresses an estimate on how much a partition could be improved if a vertex $v$ is moved to another subset $S_{n}$. Given a vertex $v$ from subset $S_{c}$, the gain $g(v, n)$ can be computed for every other subset $S_{n}, n \neq c$. The selection of the vertex with the highest gain, as well as the updates needed after each move, are achieved efficiently by using an adaptation of bucket sorting [6] that was originally proposed in [14] for graph bisection.

Let $I=\left\{S_{1}, S_{2}, \ldots, S_{k}\right\}$ be a $k$-partition, $V\left(S_{i}\right)$ the set of border vertices relative to subset $S_{i}$, and $S_{\max }=$ $\left\{S_{i} \mid \max _{i \in\{1 . . k\}}\left\{W\left(S_{i}\right)\right\}\right\}$ the subset with the maximum vertex weight. The neighborhood relations $N_{1}$ and $N_{2}$ can be explained by the two move operators given below.

Move 1: Move one highest gain vertex $v_{m}$. Choose randomly a subset $S_{m} \in\left\{S_{1}, S_{2}, \ldots, S_{k}\right\}-\left\{S_{\max }\right\}$. Then, select the highest gain vertex $v_{m} \in V\left(S_{m}\right)$ whose current subset is $S_{c}$, such that $S_{c} \in\left\{S \in I \mid W(S)>W\left(S_{m}\right)\right\}$. Move the selected vertex $v_{m}$ to subset $S_{m}$.

Move 2: Move two highest gain vertices $v_{m}$ and $v_{n}$. 


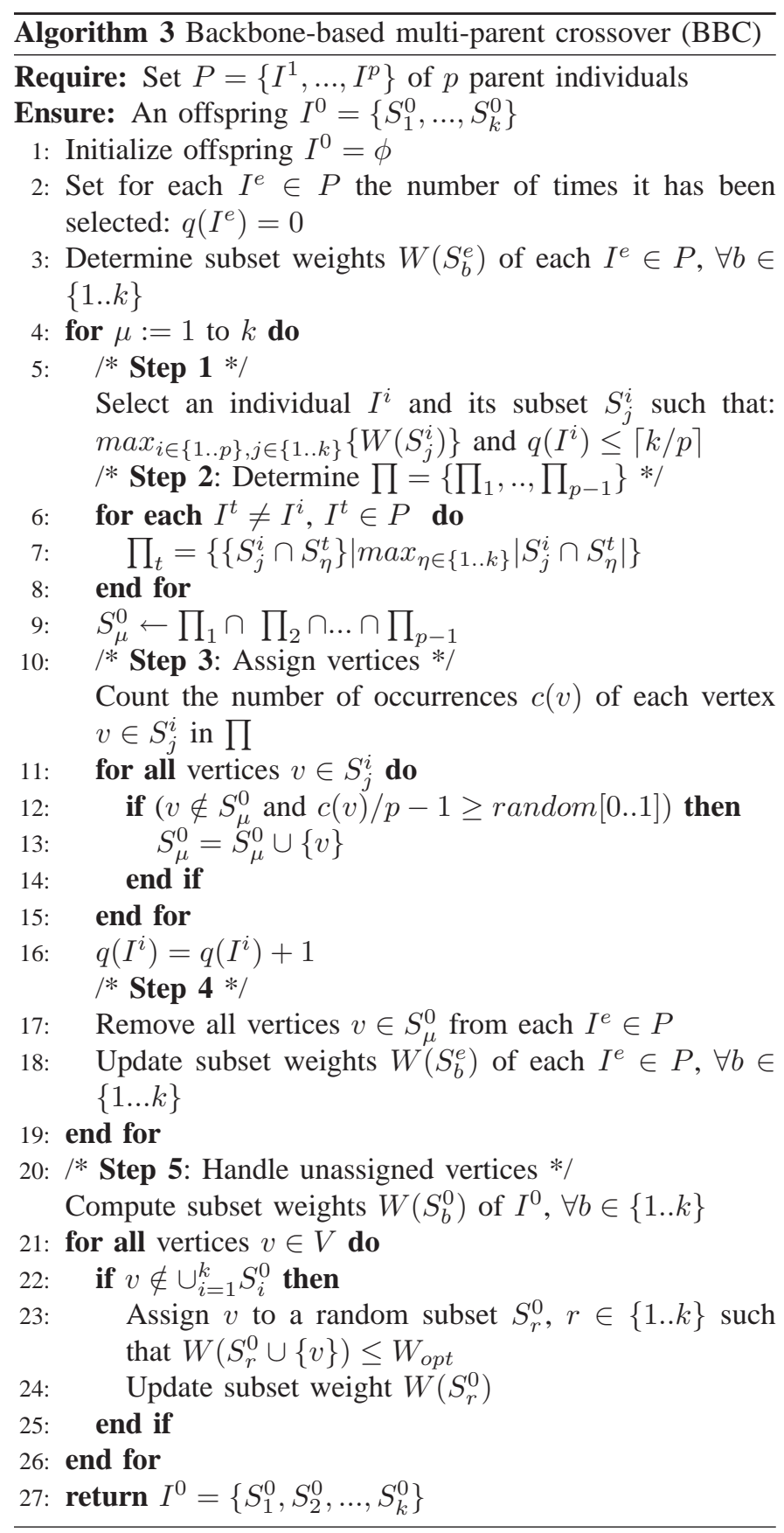

Choose vertex $v_{m}$ and its new subset $S_{m}$ as in the first move operator. Choose randomly a new subset $S_{n} \in\left\{S_{1}, S_{2}, \ldots, S_{k}\right\}-\left\{S_{\max }, S_{m}\right\}$. Then, select vertex $v_{n} \in V\left(S_{n}\right)$ whose current subset is $S_{c}$, such that $S_{c} \in\left\{S \in I \mid S \neq S_{n}\right\}$. Move $v_{m}$ to $S_{m}$, and $v_{n}$ to $S_{n}$.

It is important to note that these move operators progressively lead the search toward a balanced partition since they basically constraint (partially with Move 2) vertex migration from heavy weight subsets to light weight subsets. Indeed, with Move 1 and the first choice of Move 2, a vertex can never be moved to a subset of the highest weight. The second choice of Move 2 is allowed to bring some diversification into the search.
Let $V_{\text {cand }} \subset V\left(S_{m}\right)$ be the set of the highest gain vertices which are considered for migration to subset $S_{m}$. The selection of vertex $v$, which is moved to $S_{m}$, is based on several pieces of history information.

This selection strategy is first conditioned by the tabu status (see IV-C2). It also employs two additional criteria which are based on vertex move frequency and vertex weight. The move frequency is a long term memory that records, for each vertex $v$, the number of times $v$ has been moved to a different subset. Our usage of this frequency information penalizes moves with vertices having high frequency count, by giving priority to those that have been moved less often. If there is more than one vertex with the same move frequency in the set $V_{\text {cand }}$, we use the second criterion to distinguish them and prefer a vertex $v$ which, when moved to subset $S_{m}$, minimizes the weight difference between the target subset $S_{m}$ and the original subset $S_{c}$.

2) Tabu list and tabu tenure management: Each time a vertex $v$ is moved from a subset $S_{c}$ to another subset $S_{m}$, it is forbidden to move $v$ back to its original subset $S_{c}$ for the next $t t$ iterations (tabu tenure). The tabu tenure $t t$ of $v$ is tuned adaptively according to the number of border vertices relative to $S_{c}$,

$$
t t\left(S_{c}\right)(v)=\left|V\left(S_{c}\right)\right| * \alpha,
$$

where $\left|V\left(S_{c}\right)\right|$ is the number of border vertices relative to $S_{c}$, and $\alpha$ a parameter that takes randomly a value in the range $[0.05, \ldots, 0.2]$.

3) Perturbation mechanism: Since our local search procedure focuses its search only around border (critical) vertices, it can get trapped in a local optimum. Therefore, we periodically apply a simple perturbation which consists in moving a fixed number of vertices $\gamma$, including nonborder ones, in the following way.

Let $S_{\max }$ be the set of vertices with the maximum vertex weight, $S_{\max }=\max _{i \in\{1 . . k\}}\left\{W\left(S_{i}\right)\right\}$. Randomly select a subset $S_{m} \in\left\{S_{1}, S_{2}, \ldots, S_{k}\right\}-\left\{S_{\max }\right\}$. Then, randomly choose a vertex $v_{m}$ whose current subset is $S_{c}$, such that $S_{c} \in\left\{S \in I \mid W(S)>W\left(S_{m}\right)\right\}$. Move the selected vertex $v_{m}$ to subset $S_{m}$. This operation is repeated $\gamma$ times (perturbation strength $\gamma$ is set in this paper to $2 \%$ of the total number of vertices).

Making such moves introduces naturally more diversification into the search.

\section{Population updating based on distance}

After offspring $I^{0}$ has been obtained with the proposed multi-parent crossover operator, we improve it with the perturbation-based tabu search algorithm from Section IV-C, and then decide whether $I^{0}$ should be inserted into the population. To base this decision, our algorithm combines the ideas presented in [34] and [28] and considers both the solution quality and the distance between individuals in population. Therefore, we first formally define the notion of distance between two individuals before presenting the used replacement strategy. 
1) Distance measure: To determine the distance between two individuals $I^{A}=\left\{S_{1}^{A}, S_{2}^{A}, \ldots, S_{k}^{A}\right\}$ and $I^{B}=$ $\left\{S_{1}^{B}, S_{2}^{B}, \ldots, S_{k}^{B}\right\}$, we use the well-known set-theoretic partition distance [17] (call it $d$ ), which is the minimum number of one-move steps needed to transform $I^{A}$ to $I^{B}$, i.e. $d\left(I^{A}, I^{B}\right)=|V|-\operatorname{sim}\left(I^{A}, I^{B}\right)$, where $\operatorname{sim}\left(I^{A}, I^{B}\right)$ is the similarity function.

Given the partition encoding from Section IV-A, the similarity function $\operatorname{sim}\left(I^{A}, I^{B}\right)$ is defined as $\max _{\sigma \in \Psi} \sum_{i=1}^{k} M_{i, \sigma(i)}$, where $\Psi$ is the set of all the possible permutations of $\{1,2, . ., k\}$ and $M$ a matrix with elements $M_{i, j}=\left|S_{i}^{A} \cap S_{j}^{B}\right|$. This function $\operatorname{sim}\left(I^{A}, I^{B}\right)$, which reflects structural similarity, corresponds to the number of elements that do not need to be moved to transform $I^{A}$ to $I^{B}$.

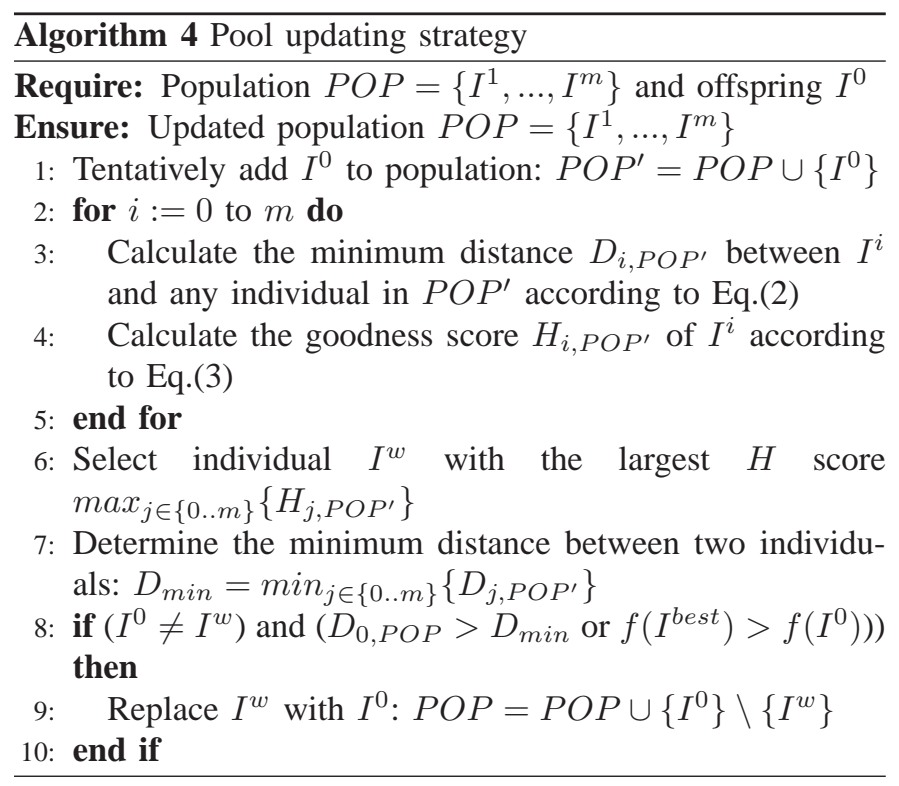

2) Pool updating strategy: Given a population $P O P=$ $\left\{I^{1}, I^{2}, \ldots, I^{m}\right\}$ of size $m$ and the distance $d_{i, j}$ between any two individuals $I^{i}$ and $I^{j}(i, j \in\{1 \ldots m\}$ and $i \neq j)$, the minimum distance between $I^{i}$ and any other individual in $P O P$ is given by:

$$
D_{i, P O P}=\min \left\{d_{i, j} \mid I^{j} \in P O P, j \neq i\right\}
$$

Offspring $I^{0}$ is then inserted into $P O P$ if it is of the best quality relative to the population, or if $D_{0, P O P}>$ $\min _{i \in\{1 . . m\}}\left\{\left(D_{i, P O P}\right)\right\}$, i.e. the minimum distance between $I^{0}$ and any other individual in the population is greater than the minimum distance between any two individuals in the population. This idea was originally proposed in [34], and has shown to be very effective in ensuring the population diversity.

To determine the individual that is to be replaced by $I^{0}$, we adopt the strategy proposed in [28]. This strategy uses a quality-and-distance scoring function $H$ to rank the individuals of the population.

$$
H_{i, P O P}=f\left(I^{i}\right)+\beta / D_{i, P O P}
$$

where $f$ is the objective function defined in Section IV-A and $\beta$ a parameter set to $\beta=0.08 *|V|$.
Our pool updating strategy consists thus of three phases: for each individual $I^{i} \in P O P$, calculating $D_{i, P O P}$ and the corresponding $H_{i, P O P}$ score (lines 2-5 of alg. 4); identifying the minimum distance $D_{\min }$ between any two individuals as well as the worst individual $I^{w}$ (lines 6-7 of alg. 4); and updating the pool (lines $8-10$ of alg. 4). This pool updating strategy contributes to maintaining a healthy diversity of the population.

\section{EXPERIMENTAL RESULTS}

\section{A. Benchmark instances}

To evaluate the efficiency of our proposed memetic approach, we carry out extensive experiments on a set of graphs that are frequently used to assess graph partitioning algorithms. These benchmark graphs are samples of small to medium scale real-life problems arising in different applications. They can be downloaded from Walshaw's Graph Partitioning Archive at: http://staffweb.cms.gre.ac.uk/ c.walshaw/partition/ in the same format as used by JOSTLE [44], METIS [25] and CHACO [18]. These graphs have unit vertex and edge weights. Table I shows the main characteristics of the graphs.

\section{B. Experimental protocol}

There is generally a trade-off between execution time and partition quality. The preference of time vs. quality is problem dependent. For instance, in the context of network layout or VLSI design, even a slight improvement of partition quality can be of significant importance. For these applications, it is worthwhile to employ a partition algorithm able to obtain excellent quality solutions even if the algorithm is computationally intensive. On the other hand, in other cases like sparse matrix-vector multiplication, a very fast algorithm is indispensable since the computing time required for the partitioning task has to be less than the time needed by a fast vector multiplication algorithm.

Our MMA algorithm is designed to produce excellent quality partitions with the possibility to be used to generate solutions of various qualities depending on the amount of computing time allowed. We thus report computational results of two experiments with short and long runs of MMA. For the first experiment, we parameterize our MMA such that each run lasts from one second to 15 minutes depending on the size of the graph (see Table II and Section V-C). The second experiment aims to assess our MMA approach with respect to the best partitions reported at the Graph Partitioning Archive. For this experiment, we use a set of parameter values that lengthens each run of the MMA algorithm (see Section V-D). The second experiment allows us to test the limit of MMA and to obtain the best results possible with more computing budgets. Given the stochastic nature of MMA, computational statistics are based on 20 or 30 independent runs of MMA on each graph.

The proposed multilevel memetic algorithm is programmed in $\mathrm{C}++$, and compiled with GNU gcc on a Xeon E5440 with $2.83 \mathrm{GHz}$ and $8 \mathrm{~GB}$. The parameter settings applied in both experiments are reported in Table II. We fix experimentally the number of parents $p$ for $\mathrm{BBC}$ relative to $k: p=3$ for $k=16 ; p=4$ for $k=2,32,64 ; p=5$ for $k=4,8$. 
TABLE I

THE LIST OF BENCHMARK GRAPHS TOGETHER WITH THEIR CHARACTERISTICS

\begin{tabular}{|c|c|c|c|c|c|c|}
\hline Grap h & $\begin{array}{l}\text { Size } \\
|V|\end{array}$ & $|E|$ & $\begin{array}{l}\text { Degr } \\
\text { Max }\end{array}$ & Min & Avg & Type \\
\hline add 20 & 2395 & 7462 & 123 & 1 & 6.23 & 20-bit adder \\
\hline data & 2851 & 15093 & 17 & 3 & 10.59 & 3D FEM \\
\hline 3elt & 4720 & 13722 & 9 & 3 & 5.81 & 2D nodal graph \\
\hline uk & 4824 & 6837 & 3 & 1 & 2.83 & 2D dual graph \\
\hline add32 & 4960 & 9462 & 31 & 1 & 3.82 & 32-bit adder \\
\hline bcsstk33 & 8738 & 291583 & 140 & 19 & 66.74 & 3D stiffness matrix \\
\hline whitaker3 & 9800 & 28989 & 8 & 3 & 5.92 & 2D nodal graph \\
\hline crack & 10240 & 30380 & 9 & 3 & 5.93 & 2D nodal graph \\
\hline wing-nodal & 10937 & 75488 & 28 & 5 & 13.80 & 3D nodal graph \\
\hline fe-4elt2 & 11143 & 32818 & 12 & 3 & 5.89 & 2D FEM \\
\hline vibrobox & 12328 & 165250 & 120 & 8 & 26.8 & Sparse matrix \\
\hline bcsstk29 & 13992 & 302748 & 70 & 4 & 43.27 & 3D stiffness matrix \\
\hline 4elt & 15606 & 45878 & 10 & 3 & 5.88 & 2D nodal graph \\
\hline fe-sphere & 16386 & 49152 & 6 & 4 & 5.99 & 3D FEM \\
\hline cti & 16840 & 48232 & 6 & 3 & 5.73 & 3D semi-structured graph \\
\hline memplus & 17758 & 54196 & 573 & 1 & 6.10 & Memory circuit \\
\hline $\operatorname{cs} 4$ & 22499 & 43858 & 4 & 2 & 3.90 & 3D nodal graph \\
\hline bcsstk30 & 28924 & 1007284 & 218 & 3 & 69.65 & 3D stiffness matrix \\
\hline bcsstk31 & 35588 & 572914 & 188 & 1 & 32.197 & 3D stiffness matrix \\
\hline fe-pwt & 36519 & 144794 & 15 & 0 & 7.93 & 3D FEL \\
\hline bcsstk32 & 44609 & 985046 & 215 & 1 & 44.1636 & 3D stiffness matrix \\
\hline fe-body & 45097 & 163734 & 28 & 0 & 7.26 & 3D FEM \\
\hline t60k & 60005 & 89440 & 3 & 2 & 2.98 & 2D dual graph \\
\hline wing & 62032 & 121544 & 4 & 2 & 2.57 & 3D dual graph \\
\hline brack2 & 62631 & 366559 & 32 & 3 & 11.71 & 3D nodal graph \\
\hline finan512 & 74752 & 261120 & 54 & 2 & 6.99 & stochastic programming matrix \\
\hline fe-tooth & 78136 & 452591 & 39 & 3 & 11.58 & 3D FEM \\
\hline fe-rotor & 99617 & 662431 & 125 & 5 & 13.30 & 3D FEM \\
\hline $598 \mathrm{a}$ & 110971 & 741934 & 26 & 5 & 13.37 & 3D FEM \\
\hline fe-ocean & 143437 & 409593 & 6 & 1 & 5.71 & 3D dual graph \\
\hline
\end{tabular}

TABLE II

SETTINGS OF IMPORTANT PARAMETERS.

\begin{tabular}{c|c|c|c}
\hline Parameters & Description & Values for Comp. 1 & Values for Comp. 2 \\
\hline$k$ & number of partition subsets & {$[2,4,8,16,32,64]$} & {$[2,4,8,16,32,64]$} \\
$P O P_{s}$ & size of population & 10 & 30 \\
$p$ & number of parents involved in crossover & {$[3,4,5]$} & {$[3,4,5]$} \\
$\lambda$ & size of tournament pool & 6 & 6 \\
$\theta$ & number of crossover operations & 10 & 30 \\
$s r$ & number of TS iter. before crossover (line 7 of alg. 1) & $|V|$ & $10 *|V|$ \\
$l r$ & number of TS iter. after crossover & $5 *|V|$ & $100 *|V|$ \\
$c t$ & coarsening threshold & 200 & 200 \\
$p_{s t r}$ & perturbation strength & $0.02 *|V|$ & $0.02 *|V|$ \\
$\gamma$ & non-improvement TS iter. before perturbation & $0.01 *|V|$ & $0.01 *|V|$ \\
\hline \multicolumn{2}{r}{}
\end{tabular}

\section{Computational results with short running time}

In this section, we show computational results of the first experiment and compare our results with those of the latest versions of METIS (METIS-4.0) [25] and CHACO (CHACO2.2) [18] available at the time of writing. For METIS, we use the multilevel pMetis algorithm, and for CHACO, we choose the multilevel KL algorithm with recursive bisection and a coarsening threshold of 100 . Notice however that the purpose of this experiment is not to show a rigorous comparison of MMA with METIS and CHACO, given that MMA is a computationally intensive stochastic algorithm while METIS and CHACO are based on very fast heuristics (order of second) whose computing time cannot be tuned. Instead, we want to assess whether MMA can obtain good partitions with a reduced running time (one second to 15 minutes). Only for this purpose, we use the results of METIS and CHACO as our references. We do not claim that MMA can be a substitute for the existing fast partition packages. Therefore, this comparison should be interpreted with caution.

The computational results of the first experiment are shown in Table III. Columns two and three report respectively the par- tition quality obtained by pMetis and $\mathrm{CHACO}$, while columns $M M A_{B}$ and $M M A_{A v}$ provide respectively the result of the best and average partition obtained with MMA (based on 30 independent runs per graph). We indicate the MMA's average partition in bold if it is better than the partitions obtained by both pMetis and CHACO. The last column shows the average time (in seconds) needed by our approach to generate the reported partition.

From Table III, we observe that the best partitions obtained with our MMA approach within a time limit ranging from less than one second up to 15 minutes are of far better quality in almost every case. In addition, the average quality of partitions obtained with MMA are also generally better than those of pMetis and CHACO.

However as $k$ increases, MMA (but also pMetis) fails to generate partitions of perfect balance in some cases. For imbalanced partitions, we indicate in parentheses the degree of imbalance or '-' if the resulting partition has an imbalance degree greater than 1.07. For these cases, partition balance can not be completely established since the tabu search procedure does not move vertices strictly from the highest to the lightest weight subsets (see Section IV-C1). Although Move 1 of the 
TABLE III

COMPARISON OF OUR MMA APPROACH WITH PMETIS AND CHACO FOR $k \in\{2,4,8,16,32,64\}$. PMETIS IS PART OF THE METIS FAMILY OF Multilevel Partitioning Algorithms. CHACO IS A PACKAGE THAT INTEGRATES A VARIETY OF ALGORITHMS FOR GRAPH PARTITIONING. BESIDE PROVIDING THE VALUES OF PARTITIONS OBTAINED WITH PMETIS AND CHACO, WE SHOW THE BEST $\left(M M A_{B}\right)$ AND AVERAGE $\left(M M A_{A v}\right)$ PARTITIONS OBTAINED WITH MMA AFTER 30 RUNS, AS WELL AS THE AVERAGE TIME IN SECONDS. IF THE PARTITION IS IMBALANCED, WE REPORT THE DEGREE OF IMBALANCE BETWEEN PARENTHESES.

\begin{tabular}{|c|c|c|c|c|c|c|c|c|c|c|c|c|c|c|c|}
\hline & \multicolumn{5}{|l|}{$\mathrm{k}=2$} & \multicolumn{5}{|l|}{$\mathrm{k}=4$} & \multicolumn{5}{|l|}{$\mathrm{k}=8$} \\
\hline Graph & pMetis & CHACO & $M M A_{B}$ & $M M A_{A v}$ & time & pMetis & CHACO & $M M A_{B}$ & $M M A_{A v}$ & time & pMetis & CHACO & $M M A_{B}$ & $M M A_{A v}$ & time \\
\hline add 20 & 729 & 742 & 697 & 709.0 & 0.9 & 1292 & 1329 & 1179 & 1205.1 & 3.6 & 1907 & 1867 & 1708 & 1730.7 & 8.4 \\
\hline data & 218 & 199 & 189 & 195.0 & 0.8 & 480 & 433 & 383 & 409.7 & 3.0 & 842 & 783 & 674 & 699.6 & 3.2 \\
\hline 3elt & 108 & 103 & 90 & 103.2 & 1.5 & 231 & 234 & 201 & 208.4 & 4.8 & 388 & 389 & 348 & 359.5 & 4.9 \\
\hline uk & 23 & 36 & 20 & 24.4 & 1.5 & 67 & 69 & 43 & 50.8 & 4.9 & 101 & 119 & 93 & 102.0 & 5.1 \\
\hline add32 & 21 & 11 & 10 & 12.8 & 1.9 & 42 & 56 & 33 & 39.5 & 7.5 & 81 & 115 & 66 & 76.9 & 7.1 \\
\hline bcsstk33 & 10205 & 10172 & 10171 & 10224.4 & 17.1 & 23131 & 23723 & 21748 & 22119.0 & 37.6 & 40070 & 39070 & 34443 & 34585.1 & 51.3 \\
\hline whitaker3 & 135 & 131 & 127 & 127.3 & 5.1 & 406 & 425 & 383 & 394.3 & 17.1 & 719 & 765 & 659 & 669.3 & 12.7 \\
\hline crack & 187 & 225 & 184 & 188.4 & 6.5 & 382 & 445 & 367 & 372.2 & 14.1 & 773 & 777 & 685 & 711.2 & 14.2 \\
\hline wing_nodal & 1820 & 1823 & 1708 & 1721.1 & 8.0 & 4000 & 4022 & 3582 & 3625.1 & 16.9 & 6070 & 6147 & 5445 & 5534.6 & 19.5 \\
\hline fe_4elt2 & 130 & 144 & 130 & 148.6 & 10.4 & 359 & 402 & 349 & 354.2 & 17.7 & 654 & 718 & 613 & 635.7 & 15.4 \\
\hline vibrobox & 12427 & 11367 & 11184 & 11404.6 & 15.1 & 21471 & 21774 & 19288 & 19664.0 & 41.5 & 28177 & 33362 & 24790 & 24975.7 & 47.3 \\
\hline bcsstk29 & 2843 & 3140 & 2843 & 3030.1 & 20.0 & 8826 & 9202 & 8495 & 8663.1 & 37.3 & 16555 & 18158 & 15760 & 16587.6 & 43.9 \\
\hline 4elt & 154 & 158 & 139 & 179.4 & 14.3 & 406 & 433 & 327 & 360.6 & 25.6 & 635 & 688 & 548 & 581.3 & 26.4 \\
\hline fe_sphere & 440 & 424 & 386 & 386.0 & 18.1 & 872 & 852 & 771 & 773.8 & 26.2 & 1330 & 1302 & 1212 & 1231.8 & 23.0 \\
\hline cti & 334 & 372 & 334 & 340.4 & 13.6 & 1113 & 1117 & 970 & 994.4 & 29.4 & 2110 & 2102 & 1801 & 1876.6 & 31.2 \\
\hline memplus & 6337 & 7549 & 5556 & 5645.4 & 29.3 & 10559 & 11535 & 9687 & 9916.6 & 63.5 & 13110 & 14265 & 12438 & 12546.3 & 73.8 \\
\hline cs4 & 414 & 517 & 374 & 379.0 & 28.6 & 1154 & 1166 & 977 & 1003.9 & 18.3 & 1746 & 1844 & 1497 & 1532.7 & 43.8 \\
\hline bcsstk30 & 6458 & 6563 & 6394 & 9664.5 & 91.6 & 17685 & 17106 & 16681 & 20002.4 & 127.7 & 36357 & 37406 & 35909 & 38441.3 & 113.9 \\
\hline bcsstk31 & 3638 & 3391 & 2767 & 3404.1 & 74.1 & 8770 & 9199 & 7699 & 8314.2 & 125.3 & 16012 & 15551 & 13465 & 15088.7 & 150.4 \\
\hline fe_pwt & 366 & 362 & 340 & 428.8 & 85.8 & 738 & 911 & 707 & 722.6 & 91.2 & 1620 & 1670 & 1452 & 1486.5 & 92.9 \\
\hline bcsstk32 & 5672 & 6137 & 4667 & 5611.8 & 170.4 & 12205 & 15704 & 9386 & 11203.7 & 175.3 & 23601 & 25719 & 21790 & 23546.6 & 208.6 \\
\hline fe_body & 311 & 1036 & 262 & 291.5 & 88.9 & 957 & 1415 & 672 & 802.6 & 188.8 & 1348 & 2277 & 1115 & 1290.5 & 137.0 \\
\hline t60k & 100 & 91 & 84 & 111.3 & 176.7 & 255 & 235 & 221 & 256.7 & 199.6 & 561 & 524 & 490 & 524.6 & 205.2 \\
\hline wing & 950 & 901 & 814 & 842.5 & 182.8 & 2086 & 1982 & 1696 & 1740.1 & 331.4 & 3205 & 3174 & 2595 & 2668.6 & 228.7 \\
\hline brack2 & 738 & 976 & 731 & 819.5 & 173.0 & 3250 & 3462 & 3087 & 3199.6 & 318.0 & 7844 & 8026 & 7246 & 7641.1 & 274.4 \\
\hline finan512 & 162 & 162 & 162 & 194.4 & 343 & 324 & 325 & 324 & 448.2 & 427.8 & 810 & 648 & 648 & 734.4 & 429.7 \\
\hline fe_tooth & 4297 & 4642 & 3822 & 4019.1 & 277.8 & 8577 & 8430 & 6941 & 7110.9 & 435.1 & 13653 & 13484 & 11688 & 11966.6 & 344.2 \\
\hline fe_rotor & 2190 & 2151 & 2098 & 2102.3 & 426.2 & 8564 & 8215 & 7310 & 7745.1 & 525.2 & 15712 & 15244 & 13026 & 13693.3 & 518.8 \\
\hline $598 \mathrm{a}$ & 2504 & 2465 & 2398 & 2405.5 & 504.8 & 8533 & 8975 & 8044 & 8240.1 & 645.7 & 17276 & 17530 & 16061 & 16524.2 & 598.5 \\
\hline \multirow[t]{2}{*}{ fe_ocean } & 505 & 499 & 464 & 647.2 & 977.4 & 2039 & 2110 & 1897 & 1910.5 & 894.6 & 4516 & 5309 & 4210 & 4313.1 & 984.1 \\
\hline & \multicolumn{5}{|l|}{$\mathrm{k}=16$} & \multicolumn{5}{|l|}{$\mathrm{k}=32$} & \multicolumn{5}{|l|}{$\mathrm{k}=64$} \\
\hline Graph & pMetis & CHACO & $M M A_{B}$ & $M M A_{A v}$ & time & pMetis & CHACO & $M M A_{B}$ & $M M A_{A v}$ & time & pMetis & CHACO & $M M A_{B}$ & $M M A_{A v}$ & time \\
\hline add 20 & 2504 & 2297 & 2113 & 2113.8 & 16.3 & - & 2684 & $2447(1.01)$ & 2439.4 & 20.6 & $3433(1.07)$ & 3349 & $3050(1.05)$ & 3068.7 & 38.5 \\
\hline data & 1370 & 1360 & 1154 & 1168.3 & 3.4 & $2060(1.01)$ & 2143 & 1859 & 1881.9 & 3.9 & $3116(1.03)$ & 3145 & - & - & - \\
\hline 3elt & 665 & 660 & 579 & 589.9 & 5.4 & 1093 & 1106 & 978 & 988.8 & 5.6 & 1710 & 1722 & 1574 & 1583.9 & 6.4 \\
\hline uk & 189 & 211 & 164 & 182.1 & 4.7 & $316(1.01)$ & 343 & $288(1.01)$ & 307.8 & 11.2 & $495(1.02)$ & 540 & 513 & 508.2 & 5.9 \\
\hline add 32 & 128 & 174 & 117 & 129.3 & 7.9 & $288(1.01)$ & 303 & $212(1.01)$ & 224.9 & 7.7 & $626(1.02)$ & 730 & 572 & 574.8 & 8.2 \\
\hline bcsstk33 & 59791 & 61890 & 55522 & 55800.7 & 54.1 & 86008 & 84613 & 78844 & 79374.2 & 80.5 & $116203(1.01$ & 1)115530 & 125407 & 125275.0 & 142.4 \\
\hline whitaker3 & 1237 & 1218 & 1101 & 1121.6 & 11.9 & 1891 & 1895 & 1727 & 1750.7 & 12.8 & $2796(1.01)$ & 2811 & 2594 & 2621.2 & 14.9 \\
\hline crack & 1255 & 1253 & 1101 & 1142.6 & 13.9 & 1890 & 1962 & 1730 & 1767.4 & 13.71 & $2847(1.01)$ & 2904 & $2609(1.01)$ & 2640.7 & 15.8 \\
\hline wing_nodal & 9290 & 9273 & 8437 & 8508.7 & 24.3 & 13237 & 13258 & 11990 & 12064.7 & 23.5 & $17899_{(1.01)}$ & 17783 & $16075(1.01)$ & 16178 & 32.9 \\
\hline fe_4elt2 & 1152 & 1135 & 1015 & 1041.8 & 15.7 & 1787 & 1796 & 1655 & 1681.8 & 15.1 & $2765(1.01)$ & 2781 & 2574 & 2585.6 & 17.5 \\
\hline vibrobox & 37441 & 43064 & 33919 & 34839.1 & 73.2 & 46112 & 51006 & 42579 & 45100.9 & 94.2 & $53764(1.01)$ & 58392 & 55189 & 54632.1 & 135.8 \\
\hline bcsstk29 & 28151 & 28629 & 24508 & 25711.3 & 51.2 & 41190 & 42935 & 36330 & 37265.4 & 57.9 & $62891(1.01)$ & 63576 & $58272(1.01)$ & 58607.5 & 93.2 \\
\hline 4elt & 1056 & 1083 & 951 & 983.3 & 24.2 & 1769 & 1766 & 1597 & 1650.5 & 27.3 & 2953 & 2921 & 2640 & 2692.9 & 28.0 \\
\hline fe_sphere & 2030 & 2037 & 1752 & 1806.3 & 26.8 & 2913 & 2920 & 2638 & 2686.7 & 24.6 & 4191 & 4151 & 3803 & 3834.5 & 29 \\
\hline $\mathrm{cti}$ & 3181 & 3083 & 2921 & 2989.6 & 29.9 & 4605 & 4532 & 4243 & 4335.3 & 28.3 & 6461 & 6334 & 6014 & 6070.8 & 35.3 \\
\hline memplus & 14942 & 16433 & 13361 & 13558.5 & 273.8 & 17303 & 17936 & 14778 & 15110.4 & 569.6 & $19140(1.01)$ & 18978 & - & - & - \\
\hline cs4 & 2538 & 2552 & 2160 & 2221.7 & 44.8 & 3579 & 3588 & 3057 & 3111.6 & 39.6 & 4791 & 4817 & 4219 & 4278.9 & 49.8 \\
\hline bcsstk30 & 77293 & 81069 & 76258 & 76954.5 & 141.9 & 131405 & 128694 & 119413 & 123824.0 & 267.3 & 191691 & 191445 & 184829 & 204726.0 & 662.3 \\
\hline bcsstk31 & 27180 & 28557 & 24934 & 26192.0 & 147.3 & 42645 & 45354 & 40742 & 41573.5 & 123.1 & 66526 & 68375 & 61778 & 63207.6 & 266.1 \\
\hline fe_pwt & 2933 & 3200 & 2839 & 2864.5 & 83.4 & 6029 & 6036 & 5783 & 5966.2 & 92.8 & 9310 & 9231 & 8532 & 8577.7 & 96.8 \\
\hline bcsstk32 & 43371 & 47829 & 38361 & 40966.4 & 214.2 & 70020 & 73377 & 64186 & 68541.7 & 406.7 & 106733 & 108855 & 101861 & 106247.0 & 711.8 \\
\hline fe_body & 2181 & 2947 & 2118 & 2201.7 & 140.4 & 3424 & 4194 & 3385 & 3516.2 & 137.4 & 5843 & 6326 & 5576 & 5683.7 & 142.5 \\
\hline t60k & 998 & 977 & 899 & 922.9 & 228.9 & 1613 & 1594 & 1488 & 1549.3 & 194.5 & 2484 & 2506 & 2331 & 2397.7 & 201.7 \\
\hline wing & 4666 & 4671 & 4076 & 4154.1 & 235.1 & 6700 & 6843 & 5896 & 6001.0 & 224.1 & 9405 & 9308 & 8065 & 8185.9 & 220.6 \\
\hline brack2 & 12655 & 13404 & 12055 & 12322.4 & 269.6 & 19786 & 20172 & 17855 & 18411.1 & 295.6 & 28872 & 29223 & 27056 & 27853.8 & 308.5 \\
\hline finan512 & 1377 & 1296 & 1296 & 1368.9 & 391.2 & 2592 & 2592 & 2592 & 2592.0 & 362.9 & 10842 & 11962 & 10764 & 10978.4 & 350.3 \\
\hline fe_tooth & 19346 & 20887 & 17857 & 18204.5 & 348.7 & 29215 & 29849 & 25787 & 26179.6 & 345.5 & 40162 & 40306 & 35864 & 36055.7 & 456.3 \\
\hline fe_rotor & 23863 & 23936 & 20694 & 21398.3 & 460.6 & 36225 & 36367 & 32034 & 33831.1 & 559.2 & 53623 & 52497 & 48518 & 50043.5 & 943.2 \\
\hline $598 \mathrm{a}$ & 28922 & 29674 & 26361 & 26807.0 & 578.0 & 44760 & 45780 & 39470 & 40244.3 & 615.5 & 64307 & 65094 & 58483 & 58985.7 & 928.9 \\
\hline fe_ocean & 9613 & 9690 & 7908 & 8206.6 & 930.8 & 14613 & 15059 & 13237 & 13571.8 & 908.7 & 23317 & 22692 & 21143 & 21554 & 1010.3 \\
\hline
\end{tabular}


tabu search procedure generally reduces the partition imbalance after each iteration, the imbalance may not be decreased after an iteration of Move 2 since the balance constraint is only partially imposed. As it can be seen from the experimental results, the balance is always established for $k \leq 16$. For larger $k$, there is generally a large number of feasible moves implying more freedom for vertex migrations. As a result, it is more difficult to establish a perfect partition balance via the two move operators.

\section{Comparisons with the best known results}

To better assess the performance of our MMA approach, we show in this section experimental results under relaxed time constraint. We prolong the running time from several minutes up to 5 hours for the largest graph (notice that the current most effective evolutionary approach by Soper et al. [38] requires computing time of up to one week to produce state-of-theart results). The main purpose of this experiment is to know whether our MMA algorithm can improve further the current best solutions.

For comparison, we use the current best partitions reported at the Walshaw's Graph Partitioning Archive. The majority of these best partitions are generated with the hybrid evolutionary algorithm presented by Soper et al. [38], which uses JOSTLE multilevel procedure as a black box. Since each run of Soper et al.'s algorithm consists of 50,000 calls to JOSTLE, this approach requires significant running time of up to one week for large graphs. Another great portion of these current best partitions are produced with NetWorks, which is a commercialized version of JOSTLE. The remaining best results are obtained with several other approaches [20], [31], [11]. Since the experimental conditions to obtain the current best results are not available, we focus on comparing solution quality based on the best objective value.

Table IV summarizes the current best results from the Graph Partitioning Archive (column 'Best') ${ }^{1}$, the best results obtained by MMA (column 'MMA') ${ }^{2}$, as well as the average and standard deviation of partitions obtained by MMA (column 'Avg(Std)'). The last row with heading 'Total' shows the number of times MMA succeeds to improve the current best partition. All the comparisons are carried out between partitions of the same balance. In most cases, the partitions of MMA are perfectly balanced (i.e. $\varepsilon=1.00$, this is the default balance). For the cases where MMA produces imbalanced partitions $(k \in\{32,64\})$, we indicate the imbalance in parentheses next to the objective value and compare the partitions with the same imbalance.

The results show that, in the case of bisection, our MMA approach succeeds to reach the same solution quality of more than two thirds of the best balanced bisections reported at the archive. It also improves the best bisection in three cases, and produces, only in four cases, bisections that are less good than the current best ones. More importantly, as $k$ increases $(4 \leq k \leq 64)$, MMA improves even $63 \%, 90 \%, 93 \%, 83 \%$

\footnotetext{
${ }^{1}$ Results retrieved in June 2010

${ }^{2}$ Our best results are available at: http://www.info.univ-angers.fr/pub/hao/ MMAbest.html
}

and $77 \%$ of the current best $k$-partitions from the archive when $k$ is equal to $4,8,16,32$ and 64 respectively.

\section{LANDSCAPE AND STRUCTURAL ANALYSIS}

In this section, we wish to obtain some insight on the search space and provide motivations for the proposed multiparent crossover operator. For this purpose, we employ the fitness distance analysis (FDA) [22], which investigates the correlation between quality (fitness) of local optima and their distances to the optimum. Additionally, we analyze structural similarity between local optima in terms of backbone size.

\section{A. Analysis protocol}

We perform the analysis on seven graph partitioning instances of different types, with the cardinal number $k$ set to 4, 8, 16 and 32. The results reported for each graph and $k$ are based on 1500 independent runs of the multilevel perturbationbased tabu search algorithm from Section IV-C, and using the distance measure introduced in Section IV-D1. Since the optimal solutions for the selected instances are not known, we use instead the best local optima found to compute fitnessdistance correlation. Table $\mathrm{V}$ contains the data to which we will be referring in the following sections.

\section{B. FDA for selected graph partitioning instances}

The fitness distance correlation (FDC) coefficient $\rho_{f d c}$ [22] is a well-known tool for landscape analysis and can provide useful indications about the problem hardness, even if such an analysis has some known shortcomings and limits. FDC estimates how closely related are the fitness and distance to the nearest optimum. For a minimization problem, if the fitness of a solution decreases with the decrease of distance from the optimum, then it would be easy to reach the target optimum for an algorithm that concentrates around the best candidate solutions found so far, since there is a "path" to the optimum via solutions with decreasing (better) fitness. A value of $\rho_{f d c}=1$ indicates perfect correlation between fitness and distance to the optimum. For correlation of $\rho_{f d c}=-1$, the fitness function is completely misleading. FDC can also be visualized with the FD plot, where the same data used for estimating $\rho_{f d c}$ is displayed graphically. Such plots have been used to estimate the distribution of local optima for a number of problems including for instance the TSP problem [7], graph bipartitioning problem [30] and flow-shop scheduling problem [35].

In column ' $\rho_{f d c}$ ' of Table $\mathrm{V}$, we report FDC coefficients $\rho_{f d c}$ for the 7 selected graphs. For illustrative purpose, FD plots of only two graphs (3elt and vibrobox) are given in Figure 3 for $k \in\{4,8,16,32\}$. To make the difference in fitness distribution more obvious, we "normalize" the actual fitness values in the FD plots by subtracting from them the best objective value.

As it can be seen from Table $\mathrm{V}$, there is a signification fitness distance correlation in many cases. However, the FDA analysis also reveals the existence of several cases among the selected instances for which there is virtually no correlation 
TABLE IV

COMPARISON WITH THE BEST RESULTS FROM THE GRAPH PARTITIONING ARCHIVE (COLUMN 'BEST') AND THE BEST RESULTS OBTAINED BY MMA (COLUMN 'MMA') OVER 20 INDEPENDENT RUNS FOR $k \in\{2,4,8,16,32\}$. COLUMN 'AVG(STD)' PROVIDES THE AVERAGE AND STANDARD DEVIATION OF PARTITIONS OBTAINED WITH MMA. IF THE PARTITION IS IMBALANCED, WE REPORT THE DEGREE OF IMBALANCE BETWEEN PARENTHESES.

\begin{tabular}{|c|c|c|c|c|c|c|c|c|c|}
\hline & \multicolumn{3}{|l|}{$\mathrm{k}=2$} & \multicolumn{3}{|l|}{$\mathrm{k}=4$} & \multicolumn{3}{|l|}{$\mathrm{k}=8$} \\
\hline Graph & Best & MMA & $\operatorname{Avg}(\operatorname{Std})$ & Best & MMA & $\operatorname{Avg}(\operatorname{Std})$ & Best & MMA & $\operatorname{Avg}(\operatorname{Std})$ \\
\hline add 20 & 596 & 678 & $708.5(20.6)$ & 1203 & 1159 & $1187.6(17.1)$ & 1714 & 1696 & $1705.5(14.1)$ \\
\hline data & 189 & 189 & $189.0(0.0)$ & 383 & 382 & $391.6(8.4)$ & 679 & 669 & $675.6(4.3)$ \\
\hline 3elt & 90 & 90 & $90.0(0.0)$ & 201 & 201 & $202.4(2.2)$ & 348 & 345 & $346.8(1.0)$ \\
\hline uk & 20 & 19 & $20.8(0.9)$ & 43 & 42 & $43.1(0.7)$ & 89 & 84 & $87.1(2.2)$ \\
\hline $\operatorname{add} 32$ & 11 & 10 & $10.3(0.46)$ & 34 & 33 & $34.8(1.89)$ & 75 & 66 & $68.9(4.0)$ \\
\hline bcsstk33 & 10171 & 10171 & $10171.0(0.0)$ & 21719 & 21730 & 22193.6 (431.8) & 34579 & 34455 & $34491.3(34.9)$ \\
\hline whitaker3 & 127 & 127 & $127.0(0.0)$ & 382 & 382 & $382.1(0.2)$ & 661 & 658 & $659.4(1.3)$ \\
\hline crack & 184 & 184 & $184.0(0.0)$ & 368 & 366 & $366.0(0.0)$ & 687 & 679 & $686.6(5.7)$ \\
\hline wing-nodal & 1707 & 1707 & $1707.8(0.4)$ & 3581 & 3578 & $3608.6(27.0)$ & 5443 & 5438 & $5481.8(42.1)$ \\
\hline fe-4elt2 & 130 & 130 & $130.0(0.0)$ & 349 & 349 & $349.0(0.0)$ & 610 & 609 & $615.8(5.6)$ \\
\hline vibrobox & 10343 & 10343 & $10984.5(265.4)$ & 19245 & 19138 & $19534.1(217.2)$ & 24715 & 24583 & $24747.8(74.7)$ \\
\hline bcsstk29 & 2843 & 2843 & $2846.0(3.3)$ & 8159 & 8475 & 8484.9 (13.4) & 14322 & 15340 & $15905.8(247.0)$ \\
\hline 4elt & 139 & 139 & $139.2(0.7)$ & 326 & 326 & $329.6(4.3)$ & 548 & 547 & $548.5(2.5)$ \\
\hline fe-sphere & 386 & 386 & $386.0(0.0)$ & 770 & 770 & $771(0.9)$ & 1193 & 1165 & $1182.2(18.3)$ \\
\hline $\mathrm{cti}$ & 334 & 334 & $334.0(0.0)$ & 963 & 955 & $970.0(5.3)$ & 1812 & 1795 & $1841.1(21.4)$ \\
\hline memplus & 5513 & 5524 & $5587.6(53.2)$ & 9643 & 9646 & 9792.5 (56.6) & 11872 & 11879 & $12040.1(132.9)$ \\
\hline $\operatorname{cs} 4$ & 371 & 371 & 374.0 (1.6) & 964 & 934 & 962.1 (14.6) & 1496 & 1455 & $1474.9(12.3)$ \\
\hline bcsstk30 & 6394 & 6394 & $6394.0(0.0)$ & 16652 & 16652 & $16856.0(220.9)$ & 34921 & 34910 & $34948.4(34.9)$ \\
\hline bcsstk31 & 2762 & 2762 & $2768.1(6.5)$ & 7469 & 7355 & $7621.6(140.2)$ & 13812 & 13370 & $13755.3(383.3)$ \\
\hline fe-pwt & 340 & 340 & $358.1(5.3)$ & 709 & 707 & 718.8 (5.7) & 1465 & 1450 & $1465.1(22.1)$ \\
\hline bcsstk32 & 4667 & 4667 & $4679.5(23.5)$ & 9492 & 9318 & $9383.4(53.0)$ & 22757 & 21119 & 22377 (786.4) \\
\hline fe-body & 262 & 262 & $262.0(0.0)$ & 703 & 624 & $661.5(13.1)$ & 1234 & 1055 & $1086.9(30.5)$ \\
\hline $\mathrm{t} 60 \mathrm{k}$ & 79 & 83 & $85.5(1.2)$ & 213 & 218 & $222.3(1.3)$ & 476 & 474 & 486.9 (11.1) \\
\hline wing & 791 & 798 & $806.4(5.3)$ & 1666 & 1644 & $1672.9(22.0)$ & 2589 & 2525 & $2564.3(24.7)$ \\
\hline brack2 & 731 & 731 & $731.0(0.0)$ & 3090 & 3084 & $3100.5(24.7)$ & 7269 & 7151 & $7268.2(104.9)$ \\
\hline finan512 & 162 & 162 & $162.0(0.0)$ & 324 & 324 & 336.2 (28.9) & 648 & 648 & $656.1(24.3)$ \\
\hline fe-tooth & 3850 & 3819 & 3876.5 (78.7) & 7142 & 6919 & 6969.1 (67.6) & 11935 & 11475 & $11680.5(173.3)$ \\
\hline fe-rotor & 2098 & 2098 & 2103.8 (10.0) & 7480 & 7277 & 7630.7 (195.5) & 13292 & 12912 & $13152.4(139.0)$ \\
\hline $598 \mathrm{a}$ & 2398 & 2398 & 2398.9 (1.0) & 8154 & 8016 & $8072.6(43.3)$ & 16884 & 15938 & $16160.2(115.6)$ \\
\hline fe-ocean & 464 & 464 & $467.6(1.2)$ & 1902 & 1895 & $1898.9(2.9)$ & 4299 & 4205 & 4233.5 (16.5) \\
\hline \multirow[t]{2}{*}{ Total } & 4 & 3 & & 4 & 19 & & 2 & 27 & \\
\hline & \multicolumn{3}{|l|}{$\mathrm{k}=16$} & \multicolumn{3}{|l|}{$\mathrm{k}=32$} & \multicolumn{3}{|l|}{$\mathrm{k}=64$} \\
\hline Graph & Best & MMA & $\operatorname{Avg}($ Std $)$ & Best & MMA & $\operatorname{Avg}(\operatorname{Std})$ & Best & MMA & $\operatorname{Avg}($ Std $)$ \\
\hline $\operatorname{add} 20$ & 2149 & 2064 & $2073.6(7.5)$ & $2493(1.03)$ & 2387(1.03) & $2402.9(8.9)$ & $3152(1.03)$ & 3021(1.03) & $3021.1(7.9)$ \\
\hline data & 1162 & 1135 & $1146(6.3)$ & $1802(1.03)$ & $1824(1.02)$ & $1836.8(7.0)$ & 2798 & - & - \\
\hline 3elt & 581 & 573 & $575.6(2.4)$ & 969(1.01) & $\mathbf{9 6 9}_{(1.01)}$ & $972.3(2.57)$ & $1564(1.01)$ & $\mathbf{1 5 5 4}(1.01)$ & $1557.2(2.2)$ \\
\hline uk & 159 & 153 & $158(2.6)$ & $\mathbf{2 5 8}(1.01)$ & $264(1.01)$ & $273.0(4.5)$ & $\mathbf{4 3 8}_{(1.01)}$ & $454(1.01)$ & $460.7(5.0)$ \\
\hline $\operatorname{add} 32$ & 121 & 117 & $122.4(5.9)$ & $\mathbf{2 1 2}(1.01)$ & $\mathbf{2 1 2}(1.01)$ & 215.4 (8.6) & 493 & 499 & $514.2(7.7)$ \\
\hline bcsstk33 & 55136 & 54763 & $55250.5(337.7)$ & 78132 & 61047 & $61984(552.3)$ & $108505(1.01)$ & $107862_{(1.01)}$ & $108144(219.7)$ \\
\hline whitaker3 & 1108 & 1095 & $1102.3(4.3)$ & 1718 & 1697 & $1708.4(4.3)$ & 2569 & 2552 & $2563.1(8.3)$ \\
\hline crack & 1108 & 1094 & $1111.4(10.3)$ & 1728 & 1693 & $1704.6(5.1)$ & $2566(1.01)$ & 2561(1.01) & 2574.3 (6.4) \\
\hline wing-nodal & 8422 & 8359 & $8404.1(29.4)$ & 12080 & 11828 & $11891.1(34.55)$ & $16134(1.01)$ & $15888(1.01)$ & $15911.1(28.7)$ \\
\hline fe-4elt2 & 1018 & 1010 & $1013.1(3.4)$ & 1657 & 1633 & $1643.8(7.3)$ & 2537 & 2519 & $2533.2(6.4)$ \\
\hline vibrobox & 32654 & 32532 & $33207.3(249.7)$ & 42187 & 40098 & $40607.2(282.0)$ & $49521(1.01)$ & $\mathbf{4 8 0 4 0}(1.01)$ & 48794 (1006.3) \\
\hline bcsstk29 & 22869 & 24106 & $25167.5(694.5)$ & 36104 & 35637 & $36100.3(239.3)$ & $57054(1.01)$ & $\mathbf{5 6 7 9 2}(1.01)$ & $57640.1(448.8)$ \\
\hline 4elt & 956 & 942 & $950.2(6.096)$ & 1592 & 1563 & $1577.7(9.5)$ & 2636 & 2596 & 2603.8 (6.4) \\
\hline fe-sphere & 1750 & 1734 & 1739.4 (3.1) & 2567 & 2542 & $2565.3(12.4)$ & 3663 & 3625 & 3655.7 (15.7) \\
\hline cti & 2909 & 2837 & 2894.5 (27.8) & 4288 & 4142 & $4200.0(33.1)$ & 5955 & 5818 & $5862.8(36.0)$ \\
\hline memplus & 13516 & 13054 & $13099.0(31.1)$ & 14634 & 14501 & $14601.6(75.5)$ & 17446 & - & - \\
\hline $\operatorname{cs} 4$ & 2206 & 2107 & $2136.8(15.1)$ & 3110 & 2938 & 2979.7 (16.5) & 4223 & 4051 & 4095.7 (19.8) \\
\hline bcsstk 30 & 72007 & 70910 & 71978.7 (411.9) & 119164 & 113788 & $115716(1030.4)$ & $\mathbf{1 7 3 9 4 5}_{(1.01)}$ & $174982(1.01)$ & $176496(1066.3)$ \\
\hline bcsstk31 & 24551 & 23807 & $24152.2(226.3)$ & 38484 & 37927 & 38432.7 (447.0) & 60724 & 58241 & $58651.4(230.4)$ \\
\hline fe-pwt & 2855 & 2838 & $2845.0(6.1)$ & 5758 & 5663 & $5693.1(24.3)$ & 8495 & 8338 & $8358.4(15.0)$ \\
\hline bcsstk32 & 38711 & 36518 & $37225.7(506.1)$ & 63856 & 60898 & $61670.1(538.7)$ & 95199 & 91863 & $93633.2(826.3)$ \\
\hline fe-body & 2057 & 1834 & $1890.6(36.0)$ & 3371 & 3060 & 3101.7 (35.1) & 5460 & 4903 & $5021.7(47.7)$ \\
\hline $\mathrm{t} 60 \mathrm{k}$ & 866 & 881 & $890.1(6.3)$ & 1440 & 1431 & $1453.5(10.6)$ & 2233 & 2260 & 2273.7 (7.9) \\
\hline wing & 4198 & 3921 & 3960.9 (27.2) & 6009 & 5643 & 5703.7 (33.2) & 8132 & 7690 & 7752.7 (33.8) \\
\hline brack2 & 12323 & 11689 & $11859.5(92.6)$ & 18229 & 17398 & $17612.7(135.8)$ & 27178 & 25997 & 26154.6 (108.6) \\
\hline finan512 & 1296 & 1296 & $1356.8(35.1)$ & 2592 & 2592 & $2592.0(0.0)$ & 10560 & 10560 & $10662.3(82.6)$ \\
\hline fe-tooth & 18382 & 17428 & $17636.3(96.3)$ & 26346 & 24985 & $25292.4(178.0)$ & 35980 & 34433 & 34688.9 (100.9) \\
\hline fe-rotor & 21241 & 20438 & 20711 (129.7) & 32783 & 31369 & $31720.5(257.7)$ & 49381 & 45984 & $46364.3(209.0)$ \\
\hline $598 \mathrm{a}$ & 26427 & 25783 & $26095.5(147.1)$ & 41538 & 38682 & $38939.2(161.1)$ & 59708 & 56260 & $56574.5(163.4)$ \\
\hline fe-ocean & 8622 & 7803 & 7944.7 (98.8) & 14277 & 12903 & 13032 (75.7) & 22301 & 20146 & $20331.3(148.1)$ \\
\hline Total & 2 & 27 & & 2 & 25 & & 5 & 23 & \\
\hline
\end{tabular}



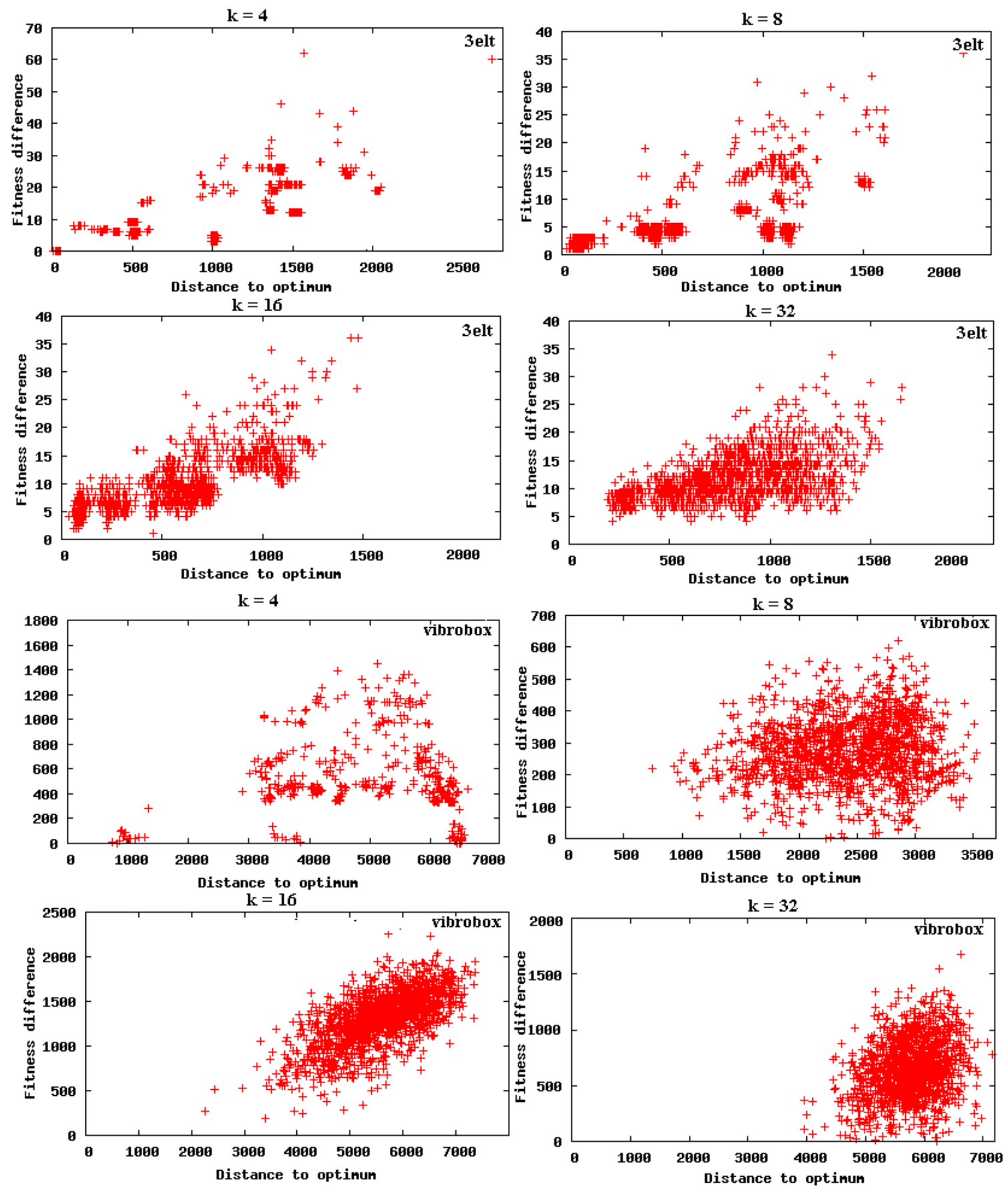

Fig. 3. FD correlation plots with respect to the normalized solution fitness and distance to the optimum for $3 e l t$ and vibrobox when $k \in\{4,8,16,32\}$. The first four plots are related to the 3elt graph, while the last four are related to the vibrobox.

between fitness and distance, i.e. cases where $\rho_{f d c}<0.15$. Indeed, from plots in Figure 3, it is clear that there is practically no correlation for 'vibrobox' when $k \in\{4,8\}$. In addition, the correlation is weak for 'vibrobox' when $k=32$. On the other hand, the strongest correlation is observed for graph '3elt' when $k \in\{4,16\}$ and 'vibrobox' when $k=16$. The presence of significant FD correlation in many cases explains to some extent why the local optimization engine (tabu search) used in MMA is extremely powerful.

The strong correlation between solution quality (fitness) and its distance to the reference solution also indicates the presence of a big valley structure in the search landscape around the selected local optimum [8]. Intuitively, in this structure the global optimum (in our case, the best local optimum) is surrounded by many local optima whose fitness values deteriorate with the increase of distance from the optimum. To investigate the existence of the big valley structure, we provide in Figure 4 plots with respect to solution fitness and average distance between any two solutions of a given set of local optima. As it can be expected, the plots give further evidence for the big valley structure in cases of graph '3elt' 

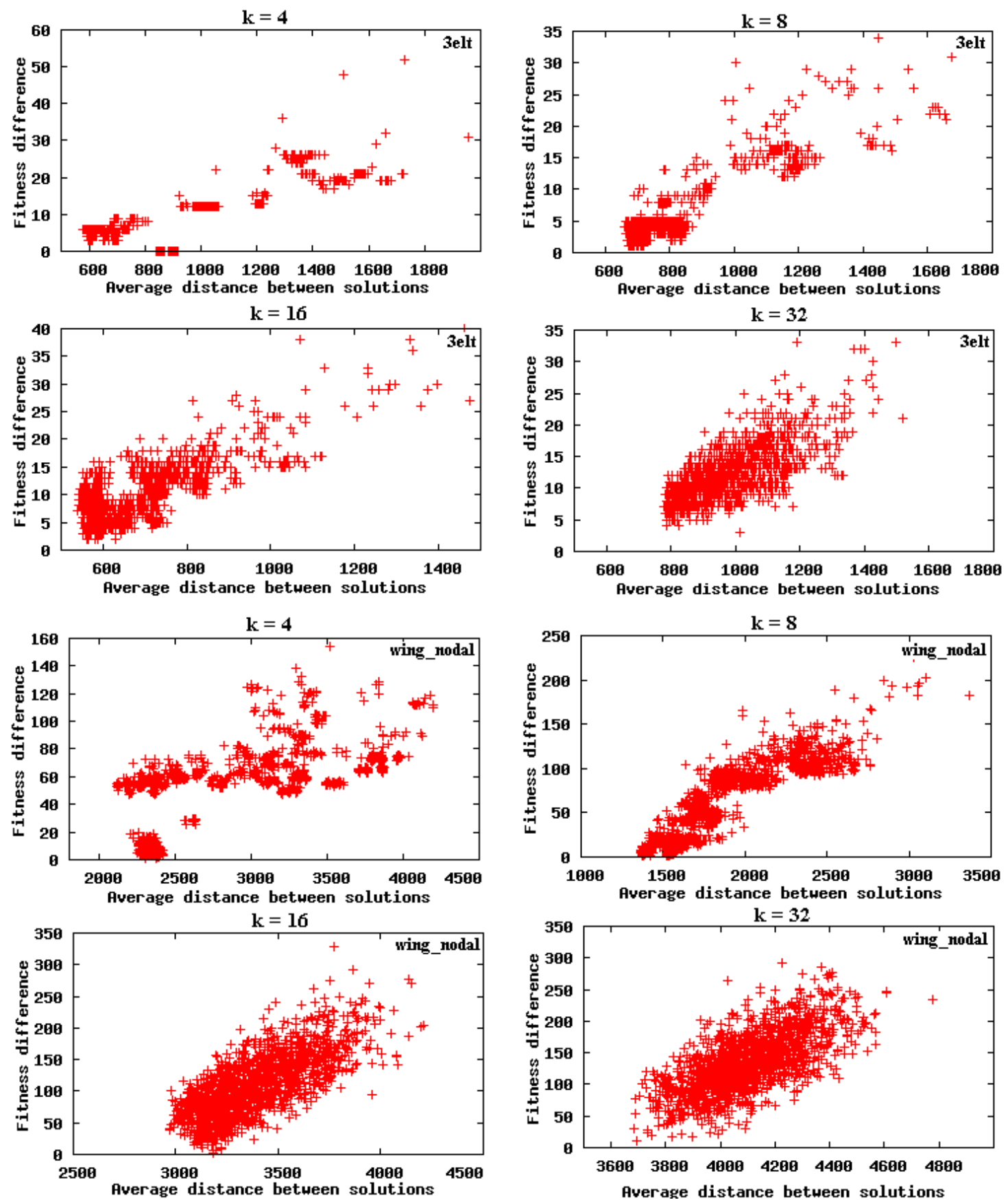

Fig. 4. FD correlation plots with respect to the normalized solution fitness and the average distance between any two solutions of a given set of local optima for 3elt and vibrobox when $k \in\{4,8,16,32\}$. The first four plots are related to 3elt, while the last four are related to the vibrobox.

and 'vibrobox' when $k=16$, i.e. high correlation between fitness and average distance between any two local optima. On the other hand, such correlation is not visible in case of 'vibrobox' when $k \in\{4,8,32\}$.

The big valley structure implies that high-quality local optima tend to be positioned centrally within the region of sampled local optima. Although we did not include fitnessdistance plots for all the analyzed graph partitioning instances, except for some very rare cases, these plots confirm our observation on the distribution of local optima in the search space.

For informative purpose, columns 'avg $d_{l o}$ ' and 'avg $d_{g o}$ ' from Table V report respectively the average distance between local optima and the average distance of local optima from the best local optimum, expressed as a percentage of $|V|$. Given that the maximum distance between any two solutions is $|V|$, these results also confirm that local optima are not uniformly distributed, but are rather concentrated within a limited number of regions in the search space.

\section{Backbone analysis and motivation for the BBC operator}

To evaluate the degree of similarity between local optima (including global optima which are technically speaking also local optima), we provide an analysis of the backbone size. 
TABLE V

ANALYTICAL RESULTS FOR SEVEN GRAPH PARTITIONING INSTANCES WHEN $k \in\{4,8,16,32\}$. COLUMNS ' $d_{l o}$ ' AND ' $d_{g o}$ ' REPORT RESPECTIVELY THE AVERAGE DISTANCE BETWEEN LOCAL OPTIMA AND THE AVERAGE DISTANCE OF LOCAL OPTIMA FROM THE BEST LOCAL OPTIMUM, EXPRESSED AS A PERCENTAGE OF $|V|$. COLUMN ' $\rho_{f d c}$ ' SHOWS THE CORRELATION COEFFICIENTS WITH RESPECT TO FITNESS AND DISTANCE, WHILE COLUMNS ' $a v g B_{l o}$ ' AND ' $B_{h q}$ ' INDICATE RESPECTIVELY (IN PERCENTAGE OF $|V|$ ) THE AVERAGE BACKBONE SIZE WITH RESPECT TO 10 RANDOMLY CHOSEN LOCAL OPTIMA, AND THE BACKBONE SIZE WITH RESPECT TO BEST LOCAL OPTIMA FOUND DURING THE SEARCH.

\begin{tabular}{|c|c|c|c|c|c|c|c|c|c|c|}
\hline \multirow[b]{2}{*}{ Graph } & \multicolumn{5}{|l|}{$\mathrm{k}=4$} & \multicolumn{5}{|l|}{$\mathrm{k}=8$} \\
\hline & avg $d_{l o}$ & $a v g d_{g o}$ & $\rho_{f d c}$ & avg $B_{l o}$ & $B_{h q}$ & $\operatorname{avg} d_{l o}$ & $\operatorname{avg} d_{g o}$ & $\rho_{f d c}$ & avg $B_{\text {lo }}$ & $B_{h q}$ \\
\hline data & 30.5 & 34.8 & 0.57 & 21.5 & 95.2 & 17.8 & 16.0 & 0.68 & 46.6 & 64.7 \\
\hline 3elt & 19.1 & 18.7 & 0.7 & 50.0 & 97.3 & 17.2 & 14.6 & 0.53 & 58.2 & 69.6 \\
\hline uk & 18 & 14.3 & 0.61 & 60.2 & 68.4 & 26.3 & 25.7 & 0.24 & 34.2 & 51.4 \\
\hline crack & 3.5 & 2.2 & 0.89 & 98.3 & 98.9 & 22.5 & 19.6 & 0.51 & 59.8 & 95.4 \\
\hline wing-nodal & 26.1 & 21.6 & 0.81 & 36.5 & 91.5 & 17.1 & 13.6 & 0.91 & 53.4 & 96.0 \\
\hline fe-4elt2 & 9.8 & 6.7 & 0.74 & 61.9 & 88.0 & 26.0 & 24.4 & 0.68 & 28.3 & 62.4 \\
\hline vibrobox & 40.1 & 41.4 & -0.02 & 21.7 & 40.7 & 22.4 & 19.7 & 0.03 & 54.1 & 52.4 \\
\hline Graph & $\begin{array}{l}\mathrm{k}=16 \\
\operatorname{avg} d_{l o}\end{array}$ & $\operatorname{avg} d_{g o}$ & $\rho_{f d c}$ & avg $B_{l o}$ & $B_{h q}$ & $\begin{array}{l}\mathrm{k}=32 \\
\operatorname{avg} d_{l o}\end{array}$ & $\operatorname{avg} d_{g o}$ & $\rho_{f d c}$ & avg $B_{l o}$ & $B_{h q}$ \\
\hline data & 22.5 & 23.7 & 0.08 & 42.3 & 42.2 & 24.9 & 23.1 & 0.6 & 32.4 & 50.9 \\
\hline 3elt & 14.0 & 12.1 & 0.75 & 61.8 & 77.2 & 20.5 & 17.1 & 0.53 & 43.3 & 61.5 \\
\hline uk & 26.9 & 25.1 & 0.33 & 32.9 & 30.0 & 27.4 & 24.9 & 0.44 & 34.6 & 61.5 \\
\hline crack & 27.7 & 22.9 & 0.74 & 24.8 & 79.6 & 28.1 & 26.3 & 0.58 & 24.5 & 33.8 \\
\hline wing-nodal & 31.0 & 27.3 & 0.56 & 25.2 & 33.2 & 37.5 & 35.6 & 0.4 & 13.5 & 15.5 \\
\hline fe-4elt2 & 16.4 & 14.7 & 0.51 & 55.3 & 73.5 & 28.7 & 25.5 & 0.51 & 20.1 & 34.3 \\
\hline vibrobox & 41.5 & 45.5 & 0.65 & 12.3 & 7.5 & 49.7 & 46.8 & 0.21 & 4.6 & 3.2 \\
\hline
\end{tabular}

While the column 'avg $B_{l o}$ ' from Table $\mathrm{V}$ reports the average backbone size with respect to 10 randomly chosen local optima, column ' $B_{h q}$ ' presents the backbone size with respect to best local optima found during the search. The backbone size is expressed as the percentage of $|V|$. From these results, we observe that except for very few cases, the backbone is generally of significant size. We also note that the values reported in ' $B_{h q}$ ' are generally higher than the ones in ' $a v g B_{l o}$ ', which indicates that the structure of a set of high quality solutions is very similar to the structure of the supposed global optimum. This suggests that if a significant number of vertices is grouped together throughout each of the high quality partitions, there is a strong chance that they are also grouped together in the global optimum. This observation constitutes the first motivation for the BBC crossover which tries to preserve the backbones through the search process.

On the other hand, the FD analysis above shows the presence of a big valley structure and high fitness-distance correlation in many cases. This provides justifications about why the tabu search based local optimization is important within our memetic approach. This additionally gives an argument for preferring a constructive crossover operator like BBC over highly destructive ones like uniform crossover. To enforce this comment, we show in Section VII a computational comparison between the BBC crossover and a uniform crossover, and study the influence of the perturbation strength within BBC.

\section{A COMPARATIVE ANALYSIS OF THE BBC OPERATOR}

\section{A. Comparison with a traditional crossover operator}

We compare the performance of the $\mathrm{BBC}$ operator with an adapted uniform crossover on the set of 30 instances of the Graph Partitioning Archive for $k \in\{4,8,16,32\}$. For the uniform crossover, each vertex in the offspring partition keeps with equal probability the subset of either parent partition, with the constraint that the subset weight in the offspring partition does not exceed $W_{\text {opt }}$ (see Section II). In addition, we reinforce the randomness of the crossover by performing some vertex swaps after the uniform crossover.
In order to highlight the role of the crossover operators, we set the number of generations to 1000 and reduce the number of tabu search iterations to $0.5 *\left|V_{m}\right|$, where $\left|V_{m}\right|$ is the number of vertices in the $m^{\text {th }}$ graph level. We execute 20 times the two versions of our multilevel memetic algorithm, i.e. with $\mathrm{BBC}$ and uniform operators, and report the results in Table VI. For each value of $k$, columns ' $\mathrm{b}$. BBC' and ' $\mathrm{b}$. UNI' report respectively the best partition obtained with our approach integrating the $\mathrm{BBC}$ and uniform crossover, while columns 'BBC $\mathrm{Avg}(\mathrm{Std})$ ' and 'UNI $\mathrm{Avg}(\mathrm{Std})$ ' report respectively the average and standard deviation of the generated partitions when BBC and uniform crossovers are employed. A lower average value is indicated in bold. In addition, we perform a statistical analysis using the Welch's $t$-test, and report in column ' $p$-value' the two tailed $p$-value over the two partition sets.

From Table VI we observe that there is no significant statistical difference between the solution sets for lower values of $k$, i.e. $k \in\{4,8\}$. However, as $k$ increases, we note that our BBC operator visibly outperforms the uniform crossover in almost each case for $k \in\{16,32\}$. One explanation is that intuitively, given the semantics of the BBC crossover, larger $k$ would favor the preservation of backbone information by $\mathrm{BBC}$ whereas the number of parts has a weak influence for the uniform crossover operator as to backbone preservation. Additionally, we compare in Table VII-A the average percentage of perturbed vertices over 20 generations with the two crossovers as well as with a variant of the BBC (see Section VII-B) for $k \in\{4,8,16,32\}$. The perturbation strength is expressed as the minimum (set-theoretic partition) distance between a resulting individual and the parents participating in the crossover. As it can be expected, the perturbation introduced by the proposed crossover is always significantly weaker than the one introduced with the uniform crossover. Moreover, the degree of perturbation introduced with the uniform crossover increases with $k$. This may constitute another explanation why the $\mathrm{BBC}$ performs better than the uniform crossover for larger values of $k \in\{16,32\}$, since according to the observations 
TABLE VI

A COMPARISON OF PARTITIONS OBTAINED WITH BBC AND UNIFORM CROSSOVER OPERATOR ON THE SET OF 30 BENCHMARK GRAPHS FOR $k \in\{4,8,16,32\}$. WE REPORT THE BEST PARTITION OBTAINED WITH BBC (B. BBC) AND UNIFORM OPERATOR (B. UNI), THE AVERAGE AND STANDARD DEVIATION OF PARTITIONS OBTAINED WITH BBC (BBC AVG(STD)) AND UNIFORM CROSSOVER (UNI AVG(STD)), AND THE $p$-VALUE BETWEEN THE TWO PARTITION SETS. IF THE PARTITION IS IMBALANCED, WE INCLUDE THE DEGREE OF IMBALANCE IN PARENTHESES.

\begin{tabular}{|c|c|c|c|c|c|c|c|c|c|c|}
\hline & $\mathrm{k}=4$ & & & & & $\mathrm{k}=8$ & & & & \\
\hline Graph & b. $\mathrm{BBC}$ & BBC Avg(Std) & b. UNI & UNI Avg(Std) & p-value & b. $\mathrm{BBC}$ & BBC Avg(Std) & b. UNI & UNI Avg(Std) & $\mathrm{p}$-value \\
\hline add 20 & 1159 & $1196.9(23.5)$ & 1154 & $\mathbf{1 1 8 8 . 8}(14.5)$ & 0.199 & 1696 & $1729.2(23.2)$ & 1698 & $\mathbf{1 7 2 5 . 0}(24.5)$ & 0.581 \\
\hline data & 383 & $419.3(21.1)$ & 395 & $410.2(10.4)$ & 0.095 & 681 & $725.9(29.7)$ & 712 & $747.9(35.6)$ & 0.041 \\
\hline 3elt & 201 & $222.6(20.9)$ & 204 & 220.5 & 0.708 & 348 & $369.6(23.0)$ & 348 & $385.2(27.1)$ & 0.057 \\
\hline uk & 43 & $49.2(6.5)$ & 45 & $48.8(4.2)$ & 0.819 & 88 & $96.0(6.8)$ & 102 & $115.4(9.6)$ & 0.000 \\
\hline $\operatorname{add} 32$ & 41 & $42.0(6.9)$ & 33 & $42.8(8.6)$ & 0.748 & 67 & $76.4(7.2)$ & 74 & $96.0(17.1)$ & 0.000 \\
\hline bcsstk33 & 21779 & $22234.5(402.4)$ & 21779 & $22536.5(605.0)$ & 0.072 & 34430 & $34689.8(471.8)$ & 34480 & 34683.0 (282.2) & 0.956 \\
\hline whitaker3 & 384 & $403.1(24.1)$ & 381 & $393.5(15.0)$ & 0.141 & 659 & $670.4(10.0)$ & 662 & $685.0(24.1)$ & 0.019 \\
\hline crack & 366 & $394.1(32.5)$ & 366 & $385.5(26.9)$ & 0.368 & 687 & $714.7(15.8)$ & 687 & $727.5(34.5)$ & 0.143 \\
\hline wing_nodal & 3582 & $3646.3(45.0)$ & 3579 & $3643.4(33.6)$ & 0.819 & 5457 & $5590.6(115.9)$ & 5454 & $5515.6(41.0)$ & 0.012 \\
\hline fe_4elt 2 & 349 & $379.8(31.4)$ & 349 & $365.1(26.3)$ & 0.117 & 608 & $644.0(28.2)$ & 614 & $640.6(16.8)$ & 0.646 \\
\hline vib̄robox & 19228 & $19795.8(336.0)$ & 19170 & $19832.8(331.6)$ & 0.728 & 24935 & $25038.7(311.2)$ & 24739 & 25011.0 (150.3) & 0.723 \\
\hline bcsstk29 & 8475 & $8613.0(280.7)$ & 8493 & $8981.2(754.7)$ & 0.052 & 15586 & $16778.7(709.3)$ & 16025 & $16945.0(554.8)$ & 0.415 \\
\hline 4elt & 326 & $364.0(24.2)$ & 326 & $366.1(22.7)$ & 0.779 & 546 & 608.0 & 570 & $690.7(66.6)$ & 0.000 \\
\hline fe_sphere & 770 & $770.3(0.6)$ & 770 & $770.8(0.9)$ & 0.047 & 1208 & $1225.5(12.6)$ & 1216 & $1242.4(22.8)$ & 0.007 \\
\hline cti & 971 & $1052.0(86.0)$ & 954 & $1028.4(87.4)$ & 0.395 & 1808 & $1927.9(62.1)$ & 1799 & $1896.9(53.8)$ & 0.100 \\
\hline memplus & 9677 & $9794.3(98.6)$ & 9628 & $9688.9(55.6)$ & 0.000 & 12476 & $12533.5(35.9)$ & 12164 & $12517.6(178.4)$ & 0.700 \\
\hline $\operatorname{cs} 4$ & 971 & $995.5(16.7)$ & 970 & $995.7(25.8)$ & 0.977 & 1502 & $1531.6(20.7)$ & 1485 & $1510.8(15.8)$ & 0.001 \\
\hline bcsstk30 & 16671 & $19802.4(4547.3)$ & 16695 & $19878.4(4598.1)$ & 0.959 & 35810 & $\mathbf{3 8 3 4 2 . 3}$ (3451.6) & 35904 & $38490.6(3533.7)$ & 0.894 \\
\hline bcsstk31 & 7642 & 8633.7 (994.1) & 7396 & $7955.0(574.1)$ & 0.013 & 13730 & 15336.7 (1272.6) & 13675 & $15447.5(1224.8)$ & 0.781 \\
\hline fe_pwt & 722 & $744.3(54.6)$ & 707 & $736.4(59.7)$ & 0.665 & 1462 & $1546.5(98.2)$ & 1453 & $1506.2(31.8)$ & 0.094 \\
\hline bcsstk32 & 9499 & $13218.8(2161.9)$ & 9312 & $10907.5(1423.9)$ & 0.000 & 21420 & $23211.9(788.3)$ & 22403 & $24198.1(1318.1)$ & 0.007 \\
\hline fe_body & 660 & $826.7(99.7)$ & 691 & $821.5(97.0)$ & 0.868 & 1079 & $1164.5(60.9)$ & 1122 & $1196.5(78.4)$ & 0.158 \\
\hline $\mathrm{t} 6 \overline{0} \mathrm{k}$ & 219 & $248.3(44.6)$ & 223 & $252.1(34.5)$ & 0.765 & 484 & $518.3(17.5)$ & 500 & $536.9(26.6)$ & 0.013 \\
\hline wing & 1665 & $1730.3(55.7)$ & 1680 & $1763.2(55.1)$ & 0.068 & 2575 & $2666.8(72.3)$ & 2629 & $2716.1(61.5)$ & 0.026 \\
\hline brack2 & 3097 & 3235.1 (208.7) & 3084 & 3275.7 (417.4) & 0.700 & 7491 & 7800.7 (207.3) & 7222 & 7756.4 (291.4) & 0.583 \\
\hline finan512 & 324 & $490.1(129.3)$ & 324 & $494.1(72)$ & 0.905 & 729 & $866.7(109.0)$ & 729 & $907.2(125.5)$ & 0.283 \\
\hline fe_tooth & 6933 & $7273.5(328.5)$ & 6975 & $7304.3(362.4)$ & 0.780 & 11875 & $12188.5(215.2)$ & 11591 & $11952.5(221.6)$ & 0.002 \\
\hline fe_rotor & 7296 & 7888.1 (419.2) & 7495 & 7932.4 (429.9) & 0.744 & 13184 & $13784.2(458.1)$ & 13396 & $14039.2(440.0)$ & 0.081 \\
\hline $598 \mathrm{a}$ & 8071 & $8352.4(313.2)$ & 8058 & $8321.7(396.2)$ & 0.787 & 16032 & $16899.1(632.6)$ & 16124 & $16888.0(670.6)$ & 0.957 \\
\hline fe_ocean & 1890 & $2007.9(270.2)$ & 1890 & $2220.0(425.5)$ & 0.069 & 4224 & $4502.3(257.2)$ & 4211 & $4389.7(149.7)$ & 0.100 \\
\hline Graph & $\begin{array}{l}k=16 \\
\text { b. BBC }\end{array}$ & BBC Avg(Std) & b. UNI & UNI $\operatorname{Avg}($ Std $)$ & $\mathrm{p}$-value & $\begin{array}{l}\mathrm{k}=32 \\
\mathrm{~b} . \mathrm{BBC}\end{array}$ & BBC $\operatorname{Avg}(\mathrm{Std})$ & b. UNI & UNI Avg(Std) & $\mathrm{p}$-value \\
\hline add 20 & 2073 & $2094.9(13.7)$ & $2145(1.01)$ & $2201.7(30.6)$ & 0.000 & $2436(1.03)$ & $2425.9(13.8)$ & $2541(1.03)$ & $2620.3(84.9)$ & 0.000 \\
\hline data & 1138 & $1164.3(18.5)$ & 1348 & $1409(39.4)$ & 0.000 & $1833(1.07)$ & $1870.0(20.7)$ & 204 & $2074.5(37.2)$ & 0.000 \\
\hline 3elt & 600 & $595.7(10.0)$ & 661 & $734.3(30.2)$ & 0.000 & 971 & $988.6(11.7)$ & 1076 & $1116.3(21.2)$ & 0.000 \\
\hline $\mathrm{uk}$ & 155 & $180.2(16.5)$ & 211 & $227.3(9.5)$ & 0.000 & 330 & $344.1(8.3)$ & $342(1.01)$ & $353.7(8.1)$ & 0.001 \\
\hline $\operatorname{add} 32$ & 123 & $134.4(8.8)$ & $172(1.01)$ & $196.9(17.5)$ & 0.000 & 215 & $238.7(26.0)$ & $275(1.01)$ & $332.5(24.3)$ & 0.000 \\
\hline bcsstk33 & 55318 & $55854.9(486.9)$ & 55411 & $56477(650.5)$ & 0.002 & 77990 & $\mathbf{7 9 3 8 0 . 1}(1013.7)$ & 80642 & $82517.8(1862.8)$ & 0.000 \\
\hline whitaker3 & 1114 & $1128.4(11.5)$ & 1204 & $1292.9(40.5)$ & 0.000 & 1862 & $1802.9(60.6)$ & 1840 & $1905.4(29.2)$ & 0.000 \\
\hline crack & 1109 & $1151.9(21.8)$ & 1301 & $1384.3(39.3)$ & 0.000 & 1907 & $1895.0(78.1)$ & 1890 & $1975.8(46.4)$ & 0.000 \\
\hline wing_nodal & 8478 & $8550.5(61.8)$ & 8832 & $8962.5(279.3)$ & 0.000 & 12291 & $12383.8(78.6)$ & 12400 & $12561.8(143.5)$ & 0.000 \\
\hline fe_4elt2 & 1025 & $1054.9(18.6)$ & 1114 & $1297.4(83.4)$ & 0.000 & 1673 & $1765.0(83.5)$ & 1833 & $1902.1(44.0)$ & 0.000 \\
\hline vib̄robox & 33613 & $35292.2(784.2)$ & 35975 & $37234.7(563.2)$ & 0.000 & 43861 & $45060.1(1090.2)$ & 44462 & $45463.8(1493.1)$ & 0.336 \\
\hline bcsstk29 & 25567 & $25849.3(705.3)$ & 24456 & $25586.9(673.4)$ & 0.236 & 36514 & $37362.3(876.1)$ & 39819 & $41205.5(748.5)$ & 0.000 \\
\hline 4 elt & 959 & $992.8(53.3)$ & 1154 & $1223.8(43.1)$ & 0.000 & 1706 & $1783.3(68.3)$ & 1789 & $1863.7(37.6)$ & 0.000 \\
\hline fe_sphere & 1734 & $1808.8(45.2)$ & 1969 & $2072.3(59.5)$ & 0.000 & 2636 & $2825.8(66.6)$ & 2826 & $2886.2(29.9)$ & 0.001 \\
\hline $\mathrm{cti}^{-1}$ & 2936 & $3020.0(54.1)$ & 2962 & $3085.9(84.1)$ & 0.024 & 4237 & $4365.4(78.3)$ & 4870 & $5025.0(88.7)$ & 0.000 \\
\hline memplus & 13733 & $14061.1(204.4)$ & 13999 & $14183.5(103.4)$ & 0.024 & $14659(1.01)$ & $14907.7(255.8)$ & - & - & - \\
\hline $\operatorname{cs} 4$ & 2178 & $2220.9(18.0)$ & 2198 & $2361.5(110.7)$ & 0.000 & 3110 & $3273.8(120.1)$ & 3347 & $3424.8(48.0)$ & 0.000 \\
\hline bcsstk30 & 76258 & $76954.5(2185.0)$ & 82533 & $87968.0(4704.3)$ & 0.000 & 124969 & 128905.8 & 131402 & $134148.5(2429.8)$ & 0.000 \\
\hline bcsstk31 & 24484 & $25796.5(849.1)$ & 26360 & $30709.5(2517.2)$ & 0.000 & 41529 & $43540.6(1173.0)$ & 43758 & $47259.5(1593.3)$ & 0.000 \\
\hline fe_pwt & 2847 & $2891.8(41.1)$ & 2869 & $3052.1(94.5)$ & 0.000 & 5872 & $6268.7(138.0)$ & 6304 & $6356.3(42.0)$ & 0.013 \\
\hline bcsstk32 & 38535 & $42422.7(3105.7)$ & 46771 & $51044.6(3010.2)$ & 0.000 & 66966 & $71105.2(2711.7)$ & 70806 & $74726.5(2168.4)$ & 0.000 \\
\hline fe_body & 1890 & $2103.2(131.7)$ & 1986 & $2222.8(135.1)$ & 0.007 & 3444 & $3532.1(64.1)$ & 3809 & $3697.8(81.4)$ & 0.000 \\
\hline $\mathrm{t} 6 \overline{0} \mathrm{k}$ & 900 & $908.5(11.8)$ & 1049 & $1122.4(49.5)$ & 0.000 & 1673 & $1702.2(37.5)$ & 1661 & $1732.1(39.3)$ & 0.019 \\
\hline wing & 4097 & $4155.6(50.8)$ & 4098 & $4653.4(150.8)$ & 0.000 & 6054 & $6560.8(242.0)$ & 6572 & $6718.7(108.9)$ & 0.013 \\
\hline brack2 & 12019 & $12259.5(234.3)$ & 13019 & $13695(574.0)$ & 0.000 & 18413 & $19588.4(541.7)$ & 19613 & $20495.7(504.5)$ & 0.000 \\
\hline finan512 & 1296 & $1458.0(88.7)$ & 1539 & $1680.8(109.9)$ & 0.000 & 2592 & $2592.0(0.0)$ & 2592 & $2592.0(0.0)$ & - \\
\hline fe_tooth & 17993 & 18342.5 (198.6) & 18337 & $19521.8(990.0)$ & 0.000 & 27333 & $27785.9(986.0)$ & 28373 & $29090.2(472.9)$ & 0.000 \\
\hline fe_rotor & 21007 & $21473.4(380.2)$ & 21392 & $24644.9(1776.4)$ & 0.000 & 34348 & $35049.2(2134.0)$ & 39322 & $40929.3(806.2)$ & 0.000 \\
\hline $598 \mathrm{a}$ & 26194 & $27014.8(359.8)$ & 26534 & $28206.1(1385.0)$ & 0.001 & 39711 & $40351.5(426.7)$ & 41713 & $46046.0(2017.5)$ & 0.000 \\
\hline fe_ocean & 7982 & $8262.4(193.9)$ & 7965 & $8215.4(169.6)$ & 0.420 & 13158 & $13880.0(447.3)$ & 13321 & $13519.8(200.6)$ & 0.003 \\
\hline
\end{tabular}


TABLE VII

Percentage of Perturbed vertices with BBC, SBBC AND Uniform CROSSOVER (UNI) FOR $k \in\{4,8,16,32\}$. For BBC AND SBBC the NUMBER OF PARENTS $p=6$, WHILE FOR UNI $p=2$.

\begin{tabular}{l|lll|lllllllll}
\hline & $\mathrm{k}=4$ & & & $\mathrm{k}=8$ & & & $\mathrm{k}=16$ & & $\mathrm{k}=32$ \\
Graph & $B B C$ & $S B B C$ & $U N I$ & $B B C$ & $S B B C$ & $U N I$ & $B B C$ & $S B B C$ & $U N I$ & $B B C$ & $S B B C$ & $U N I$ \\
\hline data & 4.64 & 48.86 & 29.40 & 4.12 & 5.89 & 30.14 & 6.89 & 40.17 & 33.70 & 14.51 & 55.32 \\
3elt & 3.79 & 14.87 & 25.50 & 4.36 & 7.59 & 29.83 & 6.65 & 21.33 & 32.25 & 11.48 & 46.89 & 41.47 \\
uk & 8.36 & 26.35 & 25.92 & 5.38 & 39.38 & 31.52 & 24.13 & 60.04 & 32.71 & 10.82 & 69.14 & 35.28 \\
crack & 3.04 & 5.27 & 25.26 & 8.10 & 9.78 & 29.58 & 6.16 & 9.46 & 32.56 & 8.38 & 60.45 & 35.01 \\
wing-nodal & 2.88 & 3.62 & 25.58 & 2.26 & 5.26 & 29.64 & 9.90 & 9.06 & 31.75 & 12.73 & 61.00 & 34.75 \\
fe-4elt2 & 5.17 & 9.58 & 25.83 & 4.92 & 51.24 & 29.94 & 6.65 & 15.65 & 32.21 & 8.80 & 71.99 & 35.14 \\
vibrobox & 2.12 & 57.77 & 25.38 & 2.90 & 6.07 & 29.58 & 31.14 & 75.16 & 32.40 & 17.20 & 94.15 & 56.26 \\
\hline
\end{tabular}

from the analysis in Section VI, a weaker form of perturbation should be used because of the generally high FDC and the marked big valley structure in the landscape.

To see the convergence behavior of the proposed algorithm, we provide in Figure 5 evolution curves of the multilevel memetic algorithm respectively with the $\mathrm{BBC}$ and uniform crossovers. The curves are plotted over 300 generations performed on each graph level $\left(G_{m}, G_{m-1}, . ., G_{0}\right)$ for graph 'data' with $k=4$ and 'fe-4elt2' when $k=32$. The $x$ axis corresponds to the current number of generations, where the first 300 generations are performed on $G_{m}$, the 300-600 generations on $G_{m-1}$, etc. The $y$-axis shows the 'normalized' average fitness value obtained by subtracting the best fitness value over 10 independent executions. Both crossover operators start the refinement on a population of the same quality at the coarsest graph $G_{m}$. One observes that for graph 'data' with $k=4$, the $\mathrm{BBC}$ is outperformed by the uniform crossover, while the situation is opposite for 'fe-4elt2' with $k=32$. From the two curves, we also note that the algorithm converges in both cases toward its best partition after approximately the same number of generations. This is partially due to the quality-and-distance based population updating strategy of our MMA which ensures a healthily diversified population.

\section{B. Perturbation strength of $B B C$ operator}

In this section, we provide an analysis on the impact of perturbation strength introduced by our new multi-parent crossover operator (BBC) by comparing the performance of $\mathrm{BBC}$ from Section IV-B2 with its slight variation (call it SBBC) which mainly differs from BBC in the degree of perturbation introduced in offspring. As explained in Section IV-B2, BBC preserves all the vertices from the backbone $B=\left\{B_{1}, \ldots, B_{k}\right\}$ with respect to $p$ parent individuals, and perturbs with a certain probability a vertex $v$ if it is not present in any subset of $B$ (see alg. 3, lines 12-13). On the other hand, SBBC consists in preserving only the vertices $v \in\left\{B_{1} \cup \ldots \cup B_{k}\right\}$ and assigning the rest of vertices to random subsets $S_{r}$ of $I^{0}$, such that $W\left(S_{r} \cup\{v\}\right) \leq W_{\text {opt }}$. Consequently, SBBC implies much stronger perturbation in $I^{0}$ than BBC.

For this analysis, we use the same set of seven benchmark instances as in Section VI. To perform the statistical analysis, we use the Welch's $t$-test on sets of solutions obtained after 50 runs of our multilevel memetic algorithm. Table VII-A shows the average perturbation degree expressed as a percentage of $|V|$, which is introduced by both $\mathrm{BBC}$ and $\mathrm{SBBC}$ when $p=$
6 for $k \in\{4,8,16,32\}$. As expected, we observe that the number of perturbed vertices is always significantly larger with SBBC than with BBC.

To analyze the difference in performance, we provide in Table VIII the $t$-value, the degree of freedom $d f$, and the two tailed $p$-value over the two solution sets generated with $\mathrm{BBC}$ and SBBC respectively. We observe that the $p$-value is extremely significant ( $p$-value $<0.001$ ) except in one case, which suggests a statistically significant difference between the two solution sets. The negative $t$-value indicates that the mean value of partitions obtained by $\mathrm{BBC}$ is, except in two cases, significantly lower that the one produced by SBBC. These results imply that $\mathrm{BBC}$ outperforms in a more pronounced way $\mathrm{SBBC}$, which suggests that a too strong perturbation introduced in offspring is not desirable. This result remains consistent with that observed when the uniform crossover is used.

\section{INFLUENCE OF LOCAL OPTIMIZATION}

In this section, we briefly analyze the contribution of local optimization to the overall performance of the memetic algorithm by comparing the proposed perturbation-based tabu search procedure [6] with its hill-climbing version. The hillclimbing procedure is basically the same as the tabu search procedure with the tabu list disabled.

We perform a statistical analysis on seven graphs with $k \in$ $\{4,8,16,32\}$ using the Welch's $t$-test on solution sets obtained after 20 independent runs. For this analysis, we set the number of generations to 500, and the number of local optimization iterations before and after crossover to $0.5 *\left|V_{i}\right|$ and $5 *\left|V_{i}\right|$ respectively, where $\left|V_{i}\right|$ is the number of vertices at the $i^{\text {th }}$ graph level.

Table IX shows the statistical result between solution sets generated with two versions of our algorithms, which integrate respectively the tabu search procedure (TS) and the hill climbing procedure (HC). For each $k \in\{4,8,16,32\}$, columns ' $t$-value', 'df' and ' $p$-value' report respectively the $t$-value, degree of freedom and $p$-value over the two solution sets. In each case, the $t$-values are negative which indicates that the proposed multilevel approach always performs better when the TS algorithm is employed. From these very small $p$-values $(<0.001)$, one concludes that the difference is statistically significant. These analytical results, along with the FD analysis shown previously, provide clear evidence that the perturbationbased tabu search procedure plays an important role in the overall performance of our multilevel memetic algorithm. 


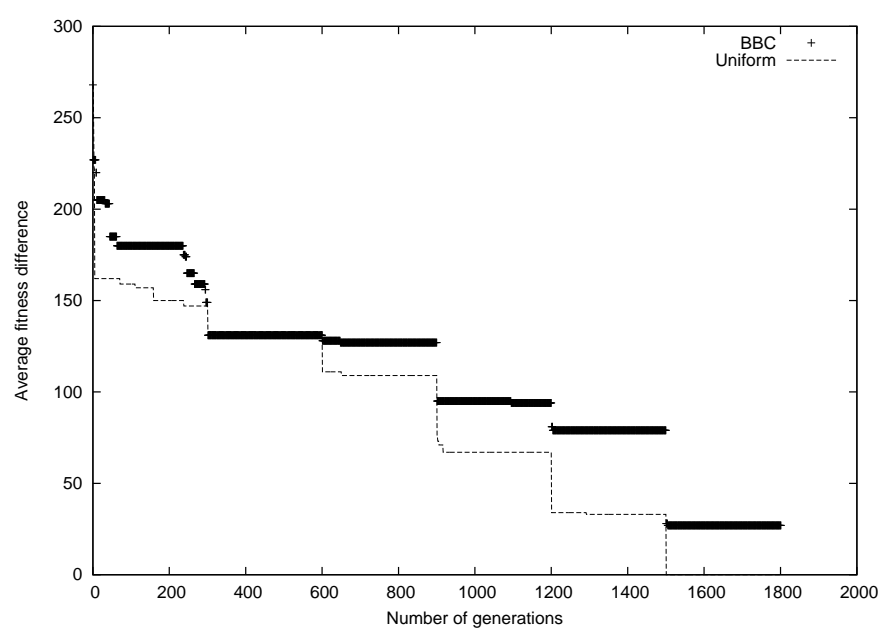

(a) Graph 'data' when $k=4$

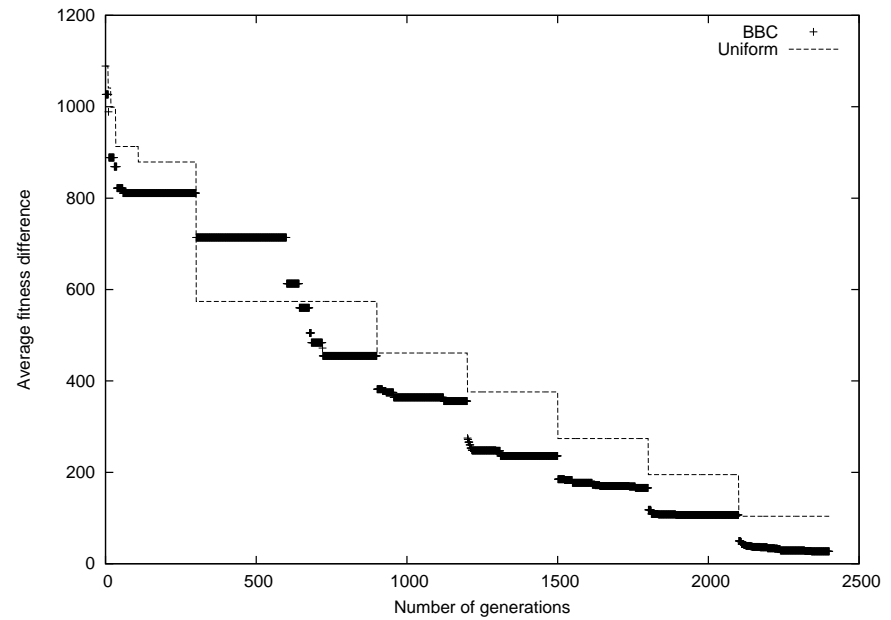

(b) Graph 'fe-4elt2' when $k=32$

Fig. 5. Evolutionary curves of the multilevel memetic algorithm with the BBC and uniform crossovers over 300 generations per graph level $G_{m}, G_{m}-1, \ldots, G_{0}$ for graphs 'data' with $k=4$ and 'fe-4elt2' with $k=32$. The $x$-axis corresponds to the current number of generations, while the $y$-axis shows the 'normalized' average fitness value over 10 independent executions. Both algorithms start with a population of the same quality.

TABLE VIII

STATISTICAL ANALYSIS USING THE WELCh's $t$-TEST OVER TWO SOLUTION SETS GENERATED WITH BBC AND SBBC WITH $p=6$ FOR $k$ EQUAL TO 4,8 , 16 AND 32. A NEGATIVE $t$-VALUE MEANS THAT BBC OUTPERFORMS THE SBBC OPERATOR.

\begin{tabular}{|c|c|c|c|c|c|c|c|c|c|c|c|c|}
\hline Graph & $\begin{array}{l}\mathrm{k}=4 \\
\text { t-value }\end{array}$ & $d f$ & $p$-value & $\begin{array}{l}\mathrm{k}=8 \\
t \text {-value }\end{array}$ & $d f$ & $p$-value & $\begin{array}{l}\mathrm{k}=16 \\
t \text {-value }\end{array}$ & $d f$ & $p$-value & $\begin{array}{l}\mathrm{k}=32 \\
\text { t-value }\end{array}$ & $d f$ & $p$-value \\
\hline data & -6.547 & 93 & 0.000 & -12.103 & 95 & 0.000 & -18.154 & 91 & 0.000 & -13.626 & 91 & 0.000 \\
\hline 3elt & -6.314 & 72 & 0.000 & -14.111 & 76 & 0.000 & -9.535 & 93 & 0.000 & -13.351 & 97 & 0.000 \\
\hline uk & -10.916 & 92 & 0.000 & -8.175 & 98 & 0.000 & -5.123 & 98 & 0.000 & -3.716 & 93 & 0.000 \\
\hline crack & -4.214 & 81 & 0.000 & -13.438 & 72 & 0.000 & -10.535 & 97 & 0.000 & -5.638 & 96 & 0.000 \\
\hline wing-nodal & 3.539 & 95 & 0.001 & 3.756 & 94 & 0.000 & -11.502 & 93 & 0.000 & -7.492 & 96 & 0.000 \\
\hline fe-4elt2 & -7.338 & 72 & 0.000 & -13.236 & 70 & 0.000 & -8.886 & 94 & 0.000 & -9.757 & 96 & 0.000 \\
\hline vibrobox & 3.390 & 98 & 0.001 & -8.410 & 84 & 0.000 & -5.165 & 92 & 0.000 & -1.103 & 98 & 0.273 \\
\hline
\end{tabular}

TABLE IX

STATISTICAL ANALYSIS USING THE WELCH's $t$-TEST OVER SOLUTION SETS GENERATED WITH TWO VERSIONS OF OUR ALGORITHMS, WHICH INTEGRATE RESPECTIVELY THE PROPOSED PERTURBATION-BASED TABU SEARCH PROCEDURE (TS) AND A HILL CLIMBING PROCEDURE (HC). A NEGATIVE $t$-VALUE MEANS THAT THE ALGORITHM WITH TS PERFORMS BETTER THAN THE ALGORITHM WITH HC.

\begin{tabular}{|c|c|c|c|c|c|c|c|c|c|c|c|c|}
\hline Graph & $\begin{array}{l}\mathrm{k}=4 \\
\text { t-value }\end{array}$ & $d f$ & $p$-value & $\begin{array}{l}\mathrm{k}=8 \\
\text { t-value }\end{array}$ & $d f$ & $p$-value & $\begin{array}{l}\mathrm{k}=16 \\
\text { t-value }\end{array}$ & $d f$ & $p$-value & $\begin{array}{l}\mathrm{k}=32 \\
\text { t-value }\end{array}$ & $d f$ & $p$-value \\
\hline data & -4.440 & 38 & 0.000 & -0.550 & 38 & 0.585 & -2.144 & 38 & 0.039 & -9.505 & 27 & 0.000 \\
\hline 3elt & -4.712 & 29 & 0.000 & -2.639 & 33 & 0.013 & -1.773 & 34 & 0.085 & -3.590 & 25 & 0.001 \\
\hline uk & -6.541 & 25 & 0.000 & -4.728 & 32 & 0.000 & -5.785 & 36 & 0.000 & -13.136 & 26 & 0.000 \\
\hline crack & -11.441 & 23 & 0.000 & -7.541 & 31 & 0.000 & -4.428 & 38 & 0.000 & -8.628 & 28 & 0.000 \\
\hline wing nodal & -4.086 & 32 & 0.000 & -3.919 & 35 & 0.000 & -9.030 & 31 & 0.000 & -10.938 & 26 & 0.000 \\
\hline fe_4elt 2 & -1.784 & 19 & 0.091 & -7.541 & 31 & 0.000 & -3.747 & 35 & 0.001 & -7.188 & 28 & 0.000 \\
\hline vibrobox & -19.047 & 37 & 0.000 & -8.222 & 21 & 0.000 & -7.232 & 32 & 0.000 & -6.693 & 27 & 0.000 \\
\hline
\end{tabular}

\section{CONCLUSION}

In this paper, we have presented a highly efficient multilevel memetic algorithm for the balanced graph partitioning problem. Our MMA algorithm uses an original backbonebased multi-parent crossover operator, a perturbation-based tabu search procedure as the local optimization engine, and a pool replacement strategy that takes into consideration both the solution quality and the distance between solutions. The backbone-based multi-parent crossover operator of MMA tries to preserve the elements which hopefully belong to the optimal partition while permitting limited perturbations within offspring solutions. The tabu search procedure (via its tabu list and occasional random moves), and the quality-and-distance based population updating strategy provide MMA with a healthy population diversity during its search.

We have proposed landscape analysis using FDC to put forward the existence of the big valley structure for some problem instances, and studied the backbone phenomenon within a set of high quality solutions which provided motivation for the design of our BBC crossover. We have also investigated the role of perturbation within the crossover operators and the influence of local optimization on the performance of the memetic algorithm.

We have assessed extensively the performance of the proposed memetic algorithm with both short and long run times, on a collection of benchmark graphs from the Graph Partitioning Archive, with the cardinal number $k$ set to 2, 4, 8, 16, 32 and 64. We have shown that the results generated in 
short computing time (from less than one second to some 15 minutes) are very competitive with those produced by the two well-known graph partitioning packages METIS and CHACO. When the running time is prolonged (from several minutes to 5 hours), our approach succeeds even to improve more than two thirds of the best partitions of the given balance reported at the Graph Partitioning Archive.

This study focuses on obtaining perfectly balanced or slightly imbalanced partitions. It is known that allowing more imbalance may lead to partitions of better quality. Indeed, when we relaxed the balance constraint up to a certain degree, with a slight modification of our MMA algorithm described in this paper, we obtained imbalanced partitions (not reported here) which are highly competitive with the best-known partitions reported in the literature. However, we believe that further research needs to be realized in order to design a dedicated algorithm which could improve even more the quality of imbalanced partitions.

\section{ACKNOWLEDGMENTS}

This work was partially supported by "Angers Loire Métropole" and the Region of "Pays de la Loire" within the Miles, Radapop and LigeRO Projects.

\section{REFERENCES}

[1] C.J. Alpert and A.B. Kahng. Recent Directions in Netlist Partitioning. Integration: the VLSI Journal, 19(12): 1-81, 1995.

[2] A. Bahreininejad, B.H.V. Topping and A.I. Khan. Finite Element Mesh Partitioning Using Neural Networks. Advances in Engineering Software, 103-115, 1996.

[3] S.T. Barnard and H.D. Simon. A Fast Multilevel Implementation of Recursive Spectral Bisection for Partitioning Unstructured Problems. Concurrency: Practice \& Experience, 6(2): 101-117, 1994.

[4] R. Battiti and A. Bertossi. Greedy and Prohibition-Based Heuristics for Graph Partitioning. IEEE Transactions on Computers, 48: 361-385, 1999.

[5] U. Benlic and J.K. Hao. An Effective Multilevel Memetic Algorithm for Balanced Graph Partitioning. Proceedings of the 22th International Conference on Tools with Artificial Intelligence, pages 121-128, 2010.

[6] U. Benlic and J.K. Hao. An Effective Multilevel Tabu Search Approach for Balanced Graph Partitioning. Computers \& Operations Research, 38(7): 1066-1075, 2011.

[7] K.D. Boese. Cost versus Distance in the Traveling Salesman Problem. Technical Report TR-950018, UCLA CS Department, 1995.

[8] K.D. Boese. Models for Iterative Global Optimization. Tech. PhD thesis, University of California, Computer Science Department, Los Angeles, CA, USA, 1996.

[9] T.N. Bui and C. Jones. A Heuristic for Reducing Fill-in in Sparse Matrix Factorization. R.F Sincovec et al. (Eds), Parallel Processing for Scientific Computing, SIAM, Philadelphia, pages 445-452, 1993.

[10] T.N. Bui and B.R. Moon. Genetic Algorithm and Graph Partitioning. IEEE Transactions on Computers, 45(7): 841-855, 1996.

[11] P. Chardaire, M. Barake, and G. P. McKeown. A PROBE based heuristic for Graph Partitioning. IEEE Transactions on Computers, 56: 17071720, 2007.

[12] M. Dell'Amico and F. Maffioli. A Tabu Search Approach to the 0-1 Equicut Problem. Meta Heuristics 1995: The State of the Art, Kluwer Academic Publishers, pages 361-377, 1996.

[13] O. Dubois and G. Dequen. A Backbone-Search Heuristic for Efficient Solving of Hard 3-SAT Formulae. Proceedings of the 17th International Joint Conference on Artificial Intelligence, pages 248-253, 2001.

[14] C. Fiduccia and R. Mattheyses. A Linear-Time Heuristics for Improving Network Partitions. Proceedings of the 19th Design Automation Conference, pages 171-185, 1982.

[15] M. Garey and D. Johnson. Computers \& Intractability: A Guide to the Theory of NP-Completeness. In W.H. Freeman and Company, 1979.
[16] C. Gil, J. Ortega, A.F. Diaz and M.G. Montoya. Annealing-Based Heuristics and Genetic Algorithms for Circuit Partitioning in Parallel Test Generation. Future Generation Computer Systems, 14(5): 439-451, 1998.

[17] D. Gusfield. Partition-Distance: A Problem and Class of Perfect Graphs Arising in Clustering. Information Processing Letters, 82(3): 159-164, 2002.

[18] B. Hendrickson and R. Leland. The Chaco User's Guide Version 2.0. Technical Report, Sandia National Laboratories, 1995.

[19] B. Hendrickson and R. Leland. A Multilevel Algorithm for Partitioning Graphs. In S. Karin. (Eds) Proceedings of Supercomputing '95, San Diego, ACM Press, New York, 1995. http://www.supercomp.org/sc95/ proceedings/509 \_BHEN/SC95.HTM

[20] M. Holtgrewe, P. Sanders, and C. Schulz. Engineering a Scalable High Quality Graph Partitioner. Technical Report, 2009.

[21] D.S. Johnson, C.R. Aragon, L.A. Mcgeoch and C. Schevon. Optimization by Simulated Annealing: An Experimental Evaluation; Part-I, Graph Partitioning. Operations Research, 37: 865-892, 1989.

[22] T. Jones and S. Forrest. Fitness Distance Correlation as a Measure of Problem Difficulty for Genetic Algorithms. Proceedings of the 6th International Conference on Genetic Algorithms, Morgan Kaufmann, pages 184-192, 1995.

[23] S. Kang and B.R. Moon. A Hybrid Genetic Algorithm for Multiway Graph Partitioning. Proceedings of the Genetic and Evolutionary Computation Conference, pages 159-166, Morgan Kaufmann, 2000.

[24] G. Karypis and V. Kumar. A Fast and High Quality Multilevel Scheme for Partitioning Irregular Graphs. SIAM Journal on Scientific Computing, 20(1): 359-392, 1998.

[25] G. Karypis and V. Kumar. Multilevel k-way Partitioning Scheme for Irregular Graphs. Journal of Parallel and Distributed Computing, 48(1): 96-129, 1998

[26] P. Kilby and J. Slaney. Backbones and Backdoors in Satisfiability. Proceedings of the 20 th American Association for Artificial Intelligence, pages $1368-1373,2005$.

[27] J.P. Kim and B.R. Moon. A Hybrid Genetic Search for Multi-way Graph Partitioning Based on Direct Partitioning. Proceedings of the Genetic and Evolutionary Computation Conference, pages 408-415, 2001.

[28] Z. Lü and J.K. Hao. A Memetic Algorithm for Graph Coloring. European Journal of Operational Research, 203(1): 241-250, 2010.

[29] N. Mansour and G.C. Fox. Allocating Data to Distributed-memory Multiprocessors by Genetic Algorithms. Concurrency: Practice \& Experience, 6(6): 485-504, 1994.

[30] P. Merz and B. Freisleben. Fitness Landscapes, Memetic Algorithms and Greedy Operators for Graph Bi-Partitioning. Evolutionary Computation, 8(1): 61-91, 2000.

[31] H. Meyerhenke, B. Monien, and T. Sauerwald. A New Diffusion-based Multilevel Algorithm for Computing Graph Partitions of Very High Quality. Journal of Parallel and Distributed Computing, 69(9): 750761, 2009.

[32] C. Papadimitriou and K. Steiglitz. Combinatorial Optimization: Algorithms and Complexity. Prentice-Hall, 1982.

[33] F. Pellegrini. Scotch and libScotch 5.1 Users Guide, INRIA, University of Bordeaux I, 2010.

[34] C.D. Porumbel, J.K. Hao, and P. Kuntz. An Evolutionaty Approach with Diversity Guarantee and Well-informed Grouping Recombination for Graph Coloring. Computers and Operations Research, 37(10): 1822$1832,2010$.

[35] C.R. Reeves. Landscapes, Operators and Heuristic Search. Annals of Operations Research, 86: 473-490, 1997.

[36] E. Rolland, H. Pirkul and F. Glover. Tabu Search for Graph Partitioning. Annals of Operations Research, 63: 209-232, 1996.

[37] J. Shi and J. Malik. Normalized Cuts and Image Segmentation. Proceedings of the IEEE Computer Society Conference on Computer Vision and Pattern Recognition, pages 731-737, 1997.

[38] A.J. Soper, C. Walshaw, and M. Cross. A Combined Evolutionary Search and Multilevel Optimisation Approach to Graph-partitioning. Journal of Global Optimization, 29(2): 225-241, 2004.

[39] A. Slowik and M. Bialko. Partitioning of VLSI Circuits on Subcircuits with Minimal Number of Connections Using Evolutionary Algorithm. In L. Rutkowski et al. (Eds.), ICAISC 2006, LNAI 4029, pages 470-478, Springer-Verlag, 2006

[40] A.G. Steenbeek, E. Marchiori, and A.E. Eiben. Finding balanced graph bi-partitions using a hybrid genetic algorithm. 1998 IEEE International Conference on Evolutionary Computation (ICEC98), pages 90-95, 1998.

[41] L. Sun and M. Leng. An Effective Refinement Algorithm Based on Swarm Intelligence for Graph Bipartitioning. International Symposium 
on Combinatorics, Algorithms, Probabilistic and Experimental Methodologie, LNCS/LNAI, pages 1-12, Springer, Heidelberg, 2007.

[42] E. Talbi and P. Bessiere. A Parallel Genetic Algorithm for the Graph Partitioning Problem. Proceedings of the 5th International Conference on Supercomputing, ACM Press, New York, pages 312-320, 1991.

[43] G. von Laszewski. Intelligent Structural Operators for the k-Way Graph Partitioning Problem. Proceedings of the 4th International Conference on Genetic Algorithms, pages 45-52, 1991.

[44] C. Walshaw and M. Cross. Mesh Partitioning : A Multilevel Balancing and Refinement Algorithm. SIAM Journal on Scientific Computing, 22(1): 63-80, 2000.

[45] C. Walshaw. Multilevel Refinement for Combinatorial Optimisation Problems. Annals of Operations Research, 131: 325-372, 2004.

[46] Y. Wang, Z. Lü, F. Glover, J.K. Hao. Backbone Guided Tabu Search for Solving the UBQP Problem, Journal of Heuristics (In Press), 2011. DOI:10.1007/s10732-011-9164-4

[47] H. Zha, X. He, C. Ding, H. Simon, M. Gu. Bipartite Graph Partitioning and Data Clustering. Proceedings of the ACM 10th International Conference on Information and Knowledge, pages 25-31, 2001.

[48] Q. Zhang, J. Sun, E.P.K. Tsang. Evolutionary Algorithm with the Guided Mutation for the Maximum Clique Problem, IEEE Transactions on Evolutionary Computation, 9(2), pp. 191-201, 2005. 